\title{
Electromagnetic structure of nucleon and Roper in soft-wall AdS/QCD
}

\author{
Thomas Gutsche, ${ }^{1}$ Valery E. Lyubovitskij, ${ }^{1,2,3,4}$ and Ivan Schmidt ${ }^{2}$ \\ ${ }^{1}$ Institut für Theoretische Physik, Universität Tübingen, Kepler Center for Astro and Particle Physics, \\ Auf der Morgenstelle 14, D-72076 Tübingen, Germany \\ ${ }^{2}$ Departamento de Física y Centro Científico Tecnológico de Valparaíso-CCTVal, \\ Universidad Técnica Federico Santa María, Casilla 110-V, Valparaíso, Chile \\ ${ }^{3}$ Department of Physics, Tomsk State University, 634050 Tomsk, Russia \\ ${ }^{4}$ Laboratory of Particle Physics, Tomsk Polytechnic University, 634050 Tomsk, Russia
}

(Received 27 December 2017; published 14 March 2018)

\begin{abstract}
We present an improved study of the electromagnetic form factors of the nucleon and of the Roper-nucleon transition using an extended version of the effective action of soft-wall AdS/QCD. We include novel contribution from additional nonminimal terms, which do not renormalize the charge and do not change the normalization of the corresponding form factors, but the inclusion of these terms results in an important contribution to the momentum dependence of the form factors and helicity amplitudes.
\end{abstract}

DOI: 10.1103/PhysRevD.97.054011

\section{INTRODUCTION}

Originally the soft-wall AdS/QCD action for the nucleon was proposed in Ref. [1]. It included a term describing the nucleon confining dynamics and the electromagnetic field, and their minimal and nonminimal couplings $Q_{N}=$ $\operatorname{diag}(1,0)$ (nucleon charge matrix) and $\eta_{N}=\operatorname{diag}\left(\eta_{p}, \eta_{n}\right)$ (nucleon matrix of anomalous magnetic moments), respectively. The use of the nonminimal couplings is essential to generate the Pauli spin-flip form factors. Later, in Ref. [2], this action was used for the calculation of generalized parton distributions of the nucleon. In Ref. [3] it was extended to take into account higher Fock states in the nucleon and additional couplings with the electromagnetic field in consistency with QCD constituent counting rules [4] for the power scaling of hadronic form factors at large values of the momentum transfer squared in the Euclidean region. In Ref. [5] soft-wall AdS/QCD was developed for the description of baryons with adjustable quantum numbers $n, J, L$, and $S$. In another development, in Refs. [6-8], the nucleon properties have been analyzed using a Hamiltonian formalism. However, their calculation of the nucleon electromagnetic properties ignored the contribution of the nonminimal coupling to the Dirac form factors, and therefore, the analysis done in Refs. [6-8], is in our opinion not fully consistent. In Ref. [7] the ideas of Ref. [6]

Published by the American Physical Society under the terms of the Creative Commons Attribution 4.0 International license. Further distribution of this work must maintain attribution to the author(s) and the published article's title, journal citation, and DOI. Funded by SCOAP ${ }^{3}$. has been extended by the inclusion of higher Fock states in the nucleon, in order to calculate nucleon electromagnetic form factors in light-front holographic QCD. In this paper the Pauli form factor is again introduced by hand, using the overlap of the $L=0$ and $L=1$ nucleon wave function. Additionally, the expression for the neutron Dirac form factor has been multiplied by hand by a free parameter $r$.

In a series of papers [9-11] we have developed a lightfront quark-diquark approach for the nucleon structure, describing nucleon parton distributions and form factors from a unified point of view. In particular, in a recent paper [11] we derived nucleon light-front wave functions, analytically matching the results of global fits to the quark parton distributions in the nucleon at the initial scale $\mu \sim 1 \mathrm{GeV}$. We also showed that the distributions obey the correct Dokshitzer-Gribov-Lipatov-Altarelli-Parisi evolution [12] to high scales. Using these constrained nucleon wave functions we get a reasonable description of data on nucleon electromagnetic form factors. We also predict the transverse parton, Wigner and Husimi distributions from a unified point of view, using our light-front wave functions and expressing them in terms of the parton distributions $q_{v}(x)$ and $\delta q_{v}(x)$. In the context of the nucleon form factors it is also important to mention a recent paper [13], where the $\gamma^{*} \rightarrow \rho$ transition form factor has been calculated in soft-wall AdS/QCD. Here it was shown that the form factor is consistent with quark counting rules for differential cross sections with single and double vector meson production. It scales as $1 / \sqrt{Q^{2}}$ and, therefore, it contributes to the electromagnetic form factors of the nucleons at subleading order. 
The nucleon resonances can be also discussed within this same formalism. E.g., the Roper resonance (the first radially excited state of the nucleon) was first considered in the context of AdS/QCD in Ref. [14], where the Dirac form factor for the electromagnetic nucleon-Roper transition was calculated in light-front holographic QCD. Later, in Ref. [15], the formalism for the study of nucleon resonances in soft-wall AdS/QCD has been developed, and the first application for a detailed description of Ropernucleon transition properties (form factors, helicity amplitudes and transition charge radii) was performed. In Refs. [16,17] the formalism proposed in [15] was used, with a different set of parameters. An overview of the application of other theoretical approaches can be found in Refs. [15,18]. This includes recent novel ideas about considering additional degrees of freedom for this state, such as a molecular nucleon-scalar $\sigma$ meson component $[19,20]$, for a realistic description of current data on Roper electroproduction performed by the CLAS Collaboration at JLab [21-23].

In the present paper we include additional nonminimal couplings of the vector field (dual to the electromagnetic field) with fermions (dual to the nucleon and Roper). Such terms do not renormalize the charge, but gives an important contribution to the momentum dependence of the nucleon and Roper-nucleon transition form factors (helicity amplitudes). The inclusion of these terms helps to improve the description of data. The paper is organized as follows. In Sec. II we briefly discuss our formalism. In Sec. III we present the analytical calculation and the numerical analysis of electromagnetic form factors and helicity amplitudes of the nucleon and the Roper. Finally, Sec. IV contains our summary and conclusions.

\section{FORMALISM}

In this section we briefly review our approach. We start with definition of the conformal metric

$$
g_{M N} x^{M} x^{N}=\epsilon_{M}^{a} \epsilon_{N}^{b} \eta_{a b} x^{M} x^{N}=\frac{1}{z^{2}}\left(d x_{\mu} d x^{\mu}-d z^{2}\right)
$$

where $\epsilon_{M}^{a}=\delta_{M}^{a} / z$ is the vielbein and $g=\left|\operatorname{det}\left(g_{M N}\right)\right|=$ $1 / z^{10}$ is the magnitude of the determinant of the $g_{M N}$.

The soft-wall AdS/QCD action $S$ for the study of the nucleon $N=(p, n)$ and Roper $\mathcal{R}=\left(\mathcal{R}_{p}, \mathcal{R}_{n}\right)$ resonance including photons is constructed in terms dual spin-1/2 fermion and vector fields, which have constrained (confined) dynamics in AdS space due to presence of background field-dilaton field $\varphi(z)=\kappa^{2} z^{2}$, where $\kappa$ is its scale parameter. The action $S$ contains a free part $S_{0}$, describing the confined dynamics of AdS fields, and interaction part $S_{\text {int }}$, describing interactions of fermions with vector field with

$$
\begin{aligned}
S= & S_{0}+S_{\text {int }}, \\
S_{0}= & \int d^{4} x d z \sqrt{g} e^{-\varphi(z)}\left\{\mathcal{L}_{N}(x, z)+\mathcal{L}_{\mathcal{R}}(x, z)\right. \\
& \left.+\mathcal{L}_{V}(x, z)\right\}, \\
S_{\text {int }}= & \int d^{4} x d z \sqrt{g} e^{-\varphi(z)}\left\{\mathcal{L}_{V N N}(x, z)+\mathcal{L}_{V \mathcal{R} R}(x, z)\right. \\
& \left.+\mathcal{L}_{V \mathcal{R} N}(x, z)\right\} .
\end{aligned}
$$

$\mathcal{L}_{N}, \mathcal{L}_{\mathcal{R}}, \mathcal{L}_{V}(x, z)$ and $\mathcal{L}_{V N N}(x, z), \mathcal{L}_{V \mathcal{R} R}(x, z), \mathcal{L}_{V \mathcal{R} N}(x, z)$ are the free and interaction Lagrangians, respectively, and are written as

$$
\begin{aligned}
\mathcal{L}_{B}(x, z) & =\sum_{i=+,-; \tau} c_{\tau}^{B} \bar{\psi}_{i, \tau}^{B}(x, z) \hat{\mathcal{D}}_{i}(z) \psi_{i, \tau}^{B}(x, z), \\
\mathcal{L}_{V}(x, z) & =-\frac{1}{4} V_{M N}(x, z) V^{M N}(x, z), \\
\mathcal{L}_{V B B}(x, z)= & \sum_{i=+,-; \tau} c_{\tau}^{B} \bar{\psi}_{i, \tau}^{B}(x, z) \hat{\mathcal{V}}_{i}^{B}(x, z) \psi_{i, \tau}^{B}(x, z), \\
\mathcal{L}_{V \mathcal{R} N}(x, z)= & \sum_{i=+,-; \tau} c_{\tau}^{\mathcal{R} N} \bar{\psi}_{i, \tau}^{\mathcal{R}}(x, z) \hat{\mathcal{V}}_{i}^{\mathcal{R} N}(x, z) \psi_{i, \tau}^{N}(x, z) \\
& + \text { H.c. },
\end{aligned}
$$

where $B=N, \mathcal{R}$ and

$$
\begin{aligned}
\hat{\mathcal{D}}_{ \pm}(z)= & \frac{i}{2} \Gamma^{M} \stackrel{\leftrightarrow}{\partial}_{M}-\frac{i}{8} \Gamma^{M} \omega_{M}^{a b}\left[\Gamma_{a}, \Gamma_{b}\right] \mp\left(\mu+U_{F}(z)\right), \\
\hat{\mathcal{V}}_{ \pm}^{H}(x, z)= & Q \Gamma^{M} V_{M}(x, z) \pm \frac{i}{4} \eta_{V}^{H}\left[\Gamma^{M}, \Gamma^{N}\right] V_{M N}(x, z) \\
& \pm \frac{i}{4} \lambda_{V}^{H} z^{2}\left[\Gamma^{M}, \Gamma^{N}\right] \partial^{K} \partial_{K} V_{M N}(x, z) \\
& \pm g_{V}^{H} \Gamma^{M} i \Gamma^{z} V_{M}(x, z)+\zeta_{V}^{H} z \Gamma^{M} \partial^{N} V_{M N}(x, z) \\
& \pm \xi_{V}^{H} z \Gamma^{M} i \Gamma^{z} \partial^{N} V_{M N}(x, z), \\
H= & N, \mathcal{R}, \mathcal{R} N .
\end{aligned}
$$

Here $\mu$ is the five-dimensional mass of the spin- $\frac{1}{2}$ AdS fermion $\mu=3 / 2+L$, with $L$ being the orbital angular momentum; $U_{F}(z)=\varphi(z)$ is the dilaton potential; $Q=\operatorname{diag}(1,0)$ is the nucleon (Roper) charge matrix; $V_{M N}=\partial_{M} V_{N}-\partial_{N} V_{M}$ is the stress tensor for the vector field; $\omega_{M}^{a b}=\left(\delta_{M}^{a} \delta_{z}^{b}-\delta_{M}^{b} \delta_{z}^{a}\right) / z$ is the spin connection term; $\sigma^{M N}=\left[\Gamma^{M}, \Gamma^{N}\right]$ is the commutator of the Dirac matrices in AdS space, which are defined as $\Gamma^{M}=\epsilon_{a}^{M} \Gamma^{a}$ and $\Gamma^{a}=\left(\gamma^{\mu},-i \gamma^{5}\right)$.

The action (2) is constructed in terms of the 5D AdS fermion fields $\psi_{ \pm, \tau}^{N}(x, z), \psi_{ \pm, \tau}^{\mathcal{R}}(x, z)$, and the vector field $V_{M}(x, z)$. Fermion fields are duals to the left- and righthanded chiral doublets of nucleons and Roper resonance $\mathcal{O}^{L}=\left(B_{1}^{L}, B_{2}^{L}\right)^{T}$ and $\mathcal{O}^{R}=\left(B_{1}^{R}, B_{2}^{R}\right)^{T}$ with $B_{1}=p, \mathcal{R}_{p}$ and $B_{2}=n, \mathcal{R}_{n}$. These fields are in the fundamental representations of the chiral $S U_{L}(2)$ and $S U_{R}(2)$ subgroups 
and are holographic analogues of the nucleon $N$ and Roper resonance $\mathcal{R}$, respectively.

The 5D AdS fields $\psi_{ \pm, \tau}^{B}(x, z)$ are products of the left/ right 4D spinor fields

$$
\psi_{n=0,1}^{L / R}(x)=\frac{1 \mp \gamma^{5}}{2} \psi_{n=0,1}(x),
$$

with spin $1 / 2$ and the bulk profiles

$$
F_{\tau, n=0,1}^{L / R}(z)=z^{2} f_{\tau, n=0,1}^{L / R}(z)
$$

with twist $\tau$ depending on the holographic (scale) variable $z$ :

$$
\begin{aligned}
& \psi_{ \pm, \tau}^{N}(x, z)=\frac{1}{\sqrt{2}}\left[\psi_{0}^{L}(x) F_{\tau, 0}^{L / R}(z) \pm \psi_{0}^{R}(x) F_{\tau, 0}^{R / L}(z)\right], \\
& \psi_{ \pm, \tau}^{\mathcal{R}}(x, z)=\frac{1}{\sqrt{2}}\left[\psi_{1}^{L}(x) F_{\tau, 1}^{L / R}(z) \pm \psi_{1}^{R}(x) F_{\tau, 1}^{R / L}(z)\right],
\end{aligned}
$$

where

$$
\begin{aligned}
& f_{\tau, 0}^{L}=\sqrt{\frac{2}{\Gamma(\tau)} \kappa^{\tau} z^{\tau-1 / 2} e^{-\kappa^{2} z^{2} / 2},} \\
& f_{\tau, 0}^{R}=\sqrt{\frac{2}{\Gamma(\tau-1)} \kappa^{\tau-1} z^{\tau-3 / 2} e^{-\kappa^{2} z^{2} / 2},} \\
& f_{\tau, 1}^{L}=\sqrt{\frac{2}{\Gamma(\tau+1)}} \kappa^{\tau} z^{\tau-1 / 2}\left(\tau-\kappa^{2} z^{2}\right) e^{-\kappa^{2} z^{2} / 2}, \\
& f_{\tau, 1}^{R}=\sqrt{\frac{2}{\Gamma(\tau)} \kappa^{\tau-1} z^{\tau-3 / 2}\left(\tau-1-\kappa^{2} z^{2}\right) e^{-\kappa^{2} z^{2} / 2} .}
\end{aligned}
$$

Here the nucleon is identified as the ground state with $n=0$ and the Roper resonance as the first radially excited state with $n=1$. In case of vector field we work in the axial gauge $V_{z}=0$ and perform a Fourier transformation of the vector field $V_{\mu}(x, z)$ with respect to the Minkowski coordinate

$$
V_{\mu}(x, z)=\int \frac{d^{4} q}{(2 \pi)^{4}} e^{i q x} V_{\mu}(q) V(q, z)
$$

We derive an EOM for the vector bulk-to-boundary propagator $V(q, z)$ dual to the $q^{2}$-dependent electromagnetic current

$$
\partial_{z}\left(\frac{e^{-\varphi(z)}}{z} \partial_{z} V(q, z)\right)+q^{2} \frac{e^{-\varphi(z)}}{z} V(q, z)=0 .
$$

The solution of this equation in terms of the gamma $\Gamma(n)$ and Tricomi $U(a, b, z)$ functions reads

$$
V(q, z)=\Gamma\left(1-\frac{q^{2}}{4 \kappa^{2}}\right) U\left(-\frac{q^{2}}{4 \kappa^{2}}, 0, \kappa^{2} z^{2}\right) .
$$

In the Euclidean region $\left(Q^{2}=-q^{2}>0\right)$ it is convenient to use the integral representation for $V(Q, z)$ [24]

$$
V(Q, z)=\kappa^{2} z^{2} \int_{0}^{1} \frac{d x}{(1-x)^{2}} x^{a} e^{-\kappa^{2} z^{2} \frac{x}{1-x}},
$$

where $x$ is the light-cone momentum fraction and $a=$ $Q^{2} /\left(4 \kappa^{2}\right)$.

The set of parameters $c_{\tau}^{N}, c_{\tau}^{\mathcal{R}}$, and $c_{\tau}^{\mathcal{R} N}$ induce mixing of the contribution of AdS fields with different twist dimension. In Refs. $[3,15]$ we showed that the parameters $c_{\tau}^{B}$ are constrained by the condition $\sum_{\tau} c_{\tau}^{B}=1$ in order to get the correct normalization of the kinetic term $\bar{\psi}_{n}(x) i \not \psi_{n}(x)$ of the four-dimensional spinor field. This condition is also consistent with electromagnetic gauge invariance.

The couplings $G_{V}^{H}=\operatorname{diag}\left(G_{V}^{H_{1}}, G_{V}^{H_{2}}\right)$, where $G=\eta, \lambda, g$, $\zeta, \xi, H_{1}=p, \mathcal{R}_{p}, \mathcal{R}_{p} p$ and $H_{2}=n, \mathcal{R}_{n}, \mathcal{R}_{n} n$ are fixed from the magnetic moments, slopes, and form factors of both the nucleon and Roper, while the couplings $c_{\tau}^{\mathcal{R} N}$ are fixed from the normalization of the Roper-nucleon helicity amplitudes. The terms proportional to the couplings $\lambda_{V}^{H}, \zeta_{V}^{H}$, and $\xi_{V}^{H}$ express novel nonminimal couplings of the fermions with the vector field. It does not renormalize the charge and does not change the corresponding form factor normalizations, but gives an important contribution to the momentum dependence of the form factors and helicity amplitudes.

The nucleon and Roper masses are identified with the expressions [3,15]

$$
M_{N}=2 \kappa \sum_{\tau} c_{\tau}^{N} \sqrt{\tau-1}, \quad M_{\mathcal{R}}=2 \kappa \sum_{\tau} c_{\tau}^{\mathcal{R}} \sqrt{\tau} .
$$

As we mentioned the set of mixing parameters $c_{\tau}^{N, \mathcal{R}}$ is constrained by the correct normalization of the kinetic term of the four-dimensional spinor field and by charge conservation as (see detail in Ref. [3]):

$$
\sum_{\tau} c_{\tau}^{N, \mathcal{R}}=1
$$

Baryon form factors are calculated analytically using bulk profiles of fermion fields and the bulk-to-boundary propagator $V(Q, z)$ of the vector field (see exact expressions in the next section). Calculation technique was described in detail in Refs. [3,15] and is briefly discussed in Appendix A).

\section{ELECTROMAGNETIC FORM FACTORS OF NUCLEON, ROPER AND ROPER-NUCLEON TRANSITIONS}

The electromagnetic form factors of the nucleon, Roper and Roper-nucleon transitions are defined by the following matrix elements, due to Lorentz and gauge invariance, 


$$
\begin{aligned}
& N \rightarrow N: M^{\mu}\left(p_{1}, \lambda_{1} ; p_{2}, \lambda_{2}\right)=\bar{u}_{N}\left(p_{2}, \lambda_{2}\right)\left[\gamma^{\mu} F_{1}^{N}\left(-q^{2}\right)-i \sigma^{\mu \nu} \frac{q_{\nu}}{2 M_{N}} F_{2}^{N}\left(-q^{2}\right)\right] u_{N}\left(p_{1}, \lambda_{1}\right), \\
& \mathcal{R} \rightarrow \mathcal{R}: M^{\mu}\left(p_{1}, \lambda_{1} ; p_{2}, \lambda_{2}\right)=\bar{u}_{\mathcal{R}}\left(p_{2}, \lambda_{2}\right)\left[\gamma^{\mu} F_{1}^{\mathcal{R}}\left(-q^{2}\right)-i \sigma^{\mu \nu} \frac{q_{\nu}}{2 M_{\mathcal{R}}} F_{2}^{\mathcal{R}}\left(-q^{2}\right)\right] u_{\mathcal{R}}\left(p_{1}, \lambda_{1}\right), \\
& \mathcal{R} \rightarrow N: M^{\mu}\left(p_{1}, \lambda_{1} ; p_{2}, \lambda_{2}\right)=\bar{u}_{N}\left(p_{2}, \lambda_{2}\right)\left[\gamma_{\perp}^{\mu} F_{1}^{\mathcal{R} N}\left(-q^{2}\right)-i \sigma^{\mu \nu} \frac{q_{\nu}}{M_{\mathcal{R}}+M_{N}} F_{2}^{\mathcal{R} N}\left(-q^{2}\right)\right] u_{\mathcal{R}}\left(p_{1}, \lambda_{1}\right),
\end{aligned}
$$

where $\gamma_{\perp}^{\mu}=\gamma^{\mu}-q^{\mu} \phi / q^{2}, q=p_{1}-p_{2}$, and $\lambda_{1}, \lambda_{2}$, and $\lambda$ are the helicities of the initial, final baryon and photon, obeying the relation $\lambda_{1}=\lambda_{2}-\lambda$.

We recall the definitions of the nucleon Sachs form factors $G_{E / M}^{N}\left(Q^{2}\right), Q^{2}=-q^{2}$ and the electromagnetic radii $\left\langle r_{E / M}^{2}\right\rangle^{N}$ in terms of the Dirac $F_{1}^{N}\left(Q^{2}\right)$ and Pauli $F_{2}^{N}\left(Q^{2}\right)$ form factors

$$
\begin{aligned}
G_{E}^{N}\left(Q^{2}\right) & =F_{1}^{N}\left(Q^{2}\right)-\frac{Q^{2}}{4 M_{N}^{2}} F_{2}^{N}\left(Q^{2}\right), \\
G_{M}^{N}\left(Q^{2}\right) & =F_{1}^{N}\left(Q^{2}\right)+F_{2}^{N}\left(Q^{2}\right), \\
\left\langle r_{E}^{2}\right\rangle^{N} & =-\left.6 \frac{d G_{E}^{N}\left(Q^{2}\right)}{d Q^{2}}\right|_{Q^{2}=0}, \\
\left\langle r_{M}^{2}\right\rangle^{N} & =-\left.\frac{6}{G_{M}^{N}(0)} \frac{d G_{M}^{N}\left(Q^{2}\right)}{d Q^{2}}\right|_{Q^{2}=0},
\end{aligned}
$$

where $G_{M}^{N}(0) \equiv \mu_{N}$ is the nucleon magnetic moment.

Now we introduce the helicity amplitudes $H_{\lambda_{2} \lambda}$, which in turn can be related to the invariant form factors $F_{i}^{\mathcal{R} N}$ (see details in Refs. [25-28]. The pertinent relation is

$$
H_{\lambda_{2} \lambda}=M_{\mu}\left(p_{1}, \lambda_{1} ; p_{2}, \lambda_{2}\right) \epsilon^{* \mu}(q, \lambda),
$$

where $\epsilon^{* \mu}(q, \lambda)$ is the polarization vector of the outgoing photon. A straightforward calculation gives [25-28]

$$
\begin{aligned}
H_{ \pm \frac{1}{2} 0} & =\sqrt{\frac{Q_{-}}{Q^{2}}}\left(F_{1}^{\mathcal{R} N} M_{+}-F_{2}^{\mathcal{R} N} \frac{Q^{2}}{M_{1}}\right), \\
H_{ \pm \frac{1}{2} \pm 1} & =-\sqrt{2 Q_{-}}\left(F_{1}^{\mathcal{R} N}+F_{2}^{\mathcal{R} N} \frac{M_{+}}{M_{1}}\right),
\end{aligned}
$$

where $M_{ \pm}=M_{1} \pm M_{2}, Q_{ \pm}=M_{ \pm}^{2}+Q^{2}$.

In the case of the Roper-nucleon transition there exists the alternative set of helicity amplitudes $\left(A_{1 / 2}, S_{1 / 2}\right)$ related to the set $\left(H_{\frac{1}{2} 0}, H_{\frac{1}{2} 1}\right)$ by [29-33]

$$
A_{1 / 2}=-b H_{\frac{1}{2} 1}, \quad S_{1 / 2}=b \frac{|\mathbf{p}|}{\sqrt{Q^{2}}} H_{\frac{1}{2} 0}
$$

where

$$
|\mathbf{p}|=\frac{\sqrt{Q_{+} Q_{-}}}{2 M_{\mathcal{R}}}, \quad b=\sqrt{\frac{\pi \alpha}{M_{+} M_{-} M_{N}}}
$$

and $\alpha=1 / 137.036$ is the fine-structure constant.

Expressions for the electromagnetic form factors of the nucleons, Roper, and Roper-nucleon transitions are given as follows:

nucleon-nucleon transition,

$$
\begin{aligned}
& F_{1}^{p}\left(Q^{2}\right)=C_{1}\left(Q^{2}\right)+g_{V}^{p} C_{2}\left(Q^{2}\right)+\eta_{V}^{p} C_{3}\left(Q^{2}\right)+\lambda_{V}^{p} C_{4}\left(Q^{2}\right)+\zeta_{V}^{p} C_{5}\left(Q^{2}\right)+\xi_{V}^{p} C_{6}\left(Q^{2}\right), \\
& F_{1}^{n}\left(Q^{2}\right)=g_{V}^{n} C_{2}\left(Q^{2}\right)+\eta_{V}^{n} C_{3}\left(Q^{2}\right)+\lambda_{V}^{n} C_{4}\left(Q^{2}\right)+\zeta_{V}^{n} C_{5}\left(Q^{2}\right)+\xi_{V}^{n} C_{6}\left(Q^{2}\right), \\
& F_{2}^{p}\left(Q^{2}\right)=\eta_{V}^{p} C_{7}\left(Q^{2}\right)+\lambda_{V}^{p} C_{8}\left(Q^{2}\right), \\
& F_{2}^{n}\left(Q^{2}\right)=\eta_{V}^{n} C_{7}\left(Q^{2}\right)+\lambda_{V}^{n} C_{8}\left(Q^{2}\right) .
\end{aligned}
$$

Roper-nucleon transition,

$$
\begin{aligned}
& F_{1}^{\mathcal{R}_{p} p}\left(Q^{2}\right)=D_{1}\left(Q^{2}\right)+g_{V}^{\mathcal{R}_{p} p} D_{2}\left(Q^{2}\right)+\eta_{V}^{\mathcal{R}_{p} p} D_{3}\left(Q^{2}\right)+\lambda_{V}^{\mathcal{R}_{p} p} D_{4}\left(Q^{2}\right)+\zeta_{V}^{\mathcal{R}_{p} p} D_{5}\left(Q^{2}\right)+\xi_{V}^{\mathcal{R}_{p} p} D_{6}\left(Q^{2}\right), \\
& F_{1}^{\mathcal{R}_{n} n}\left(Q^{2}\right)=g_{V}^{\mathcal{R}_{n} n} D_{2}\left(Q^{2}\right)+\eta_{V}^{\mathcal{R}_{n} n} D_{3}\left(Q^{2}\right)+\lambda_{V}^{\mathcal{R}_{n} n} D_{4}\left(Q^{2}\right)+\zeta_{V}^{\mathcal{R}_{n} n} D_{5}\left(Q^{2}\right)+\xi_{V}^{\mathcal{R}_{n} n} D_{6}\left(Q^{2}\right), \\
& F_{2}^{\mathcal{R}_{p} p}\left(Q^{2}\right)=\eta_{V}^{\mathcal{R}_{p} p} D_{7}\left(Q^{2}\right)+\lambda_{V}^{\mathcal{R}_{p} p} D_{8}\left(Q^{2}\right), \\
& F_{2}^{\mathcal{R}_{n} n}\left(Q^{2}\right)=\eta_{V}^{\mathcal{R}_{n} n} D_{7}\left(Q^{2}\right)+\lambda_{V}^{\mathcal{R}_{n} n} D_{8}\left(Q^{2}\right) .
\end{aligned}
$$


Roper-Roper transition,

$$
\begin{aligned}
& F_{1}^{\mathcal{R}_{p}}\left(Q^{2}\right)=E_{1}\left(Q^{2}\right)+g_{V}^{\mathcal{R}_{p}} E_{2}\left(Q^{2}\right)+\eta_{V}^{\mathcal{R}_{p}} E_{3}\left(Q^{2}\right)+\lambda_{V}^{\mathcal{R}_{p}} E_{4}\left(Q^{2}\right)+\zeta_{V}^{\mathcal{R}_{p}} E_{5}\left(Q^{2}\right)+\xi_{V}^{\mathcal{R}_{p}} E_{6}\left(Q^{2}\right), \\
& F_{1}^{\mathcal{R}_{n}}\left(Q^{2}\right)=g_{V}^{\mathcal{R}_{n}} E_{2}\left(Q^{2}\right)+\eta_{V}^{\mathcal{R}_{n}} E_{3}\left(Q^{2}\right)+\lambda_{V}^{\mathcal{R}_{n}} E_{4}\left(Q^{2}\right)+\zeta_{V}^{\mathcal{R}_{n}} E_{5}\left(Q^{2}\right)+\xi_{V}^{\mathcal{R}_{n}} E_{6}\left(Q^{2}\right), \\
& F_{2}^{\mathcal{R}_{p}}\left(Q^{2}\right)=\eta_{V}^{\mathcal{R}_{p}} E_{7}\left(Q^{2}\right)+\lambda_{V}^{\mathcal{R}_{p}} E_{8}\left(Q^{2}\right), \\
& F_{2}^{\mathcal{R}_{n}}\left(Q^{2}\right)=\eta_{V}^{\mathcal{R}_{n}} E_{7}\left(Q^{2}\right)+\lambda_{V}^{\mathcal{R}_{n}} E_{8}\left(Q^{2}\right) .
\end{aligned}
$$

The structure integrals $C_{i}\left(Q^{2}\right), D_{i}\left(Q^{2}\right)$, and $E_{i}\left(Q^{2}\right)$ are given by the analytical expressions (see in Appendix $\mathrm{B}$ ). All calculated form factors are consistent with QCD constituent counting rules [4] for the power scaling of hadronic form factors at large values of the momentum transfer squared in the Euclidean region. Note, the expressions for the transition form factors are generated by the effective action (2) with taking into account of decomposition of electromagnetic matrix elements on relativistic form factors (15) and using calculation technique (see Refs. $[3,15]$ and Appendix A). In case of nucleon-nucleon and Roper-nucleon transitions form factors contain the pieces calculated before in Refs. [3,15] and new ones, generated by the novel terms in effective action and proportional to the couplings $\lambda_{V}^{N}, \zeta_{V}^{N}, \xi_{V}^{N}$. The results for the Roper-Roper transition form factors are derived here for the first time.

The parameters, which will be used in the numerical evaluations, are fixed as follows: we use the universal dilaton parameter of $\kappa=383 \mathrm{MeV}$ fixed before in Refs. [3,15], the sets of twist mixing parameters are fixed from data on masses of nucleon

$$
c_{3}^{N}=1.800, \quad c_{4}^{N}=-1.042, \quad c_{5}^{N}=0.242
$$

and Roper

$c_{3}^{\mathcal{R}}=0.820, \quad c_{4}^{\mathcal{R}}=-0.242, \quad c_{5}^{\mathcal{R}}=0.422$.

At fixed $\kappa=383 \mathrm{MeV}$ and baryon masses $M_{N}=$ 938.27 MeV and $M_{\mathcal{R}}=1440 \mathrm{MeV}$ only two parameters from the set of six twist mixing parameters are free. E.g., parameters $c_{4}^{N, \mathcal{R}}$ and $c_{5}^{N, \mathcal{R}}$ can be fixed through the parameters $c_{3}^{N, \mathcal{R}}$ and ratios $M_{N, \mathcal{R}}$ using the matching conditions (13) and (14). The parameters $\eta_{V}^{p}=0.2988$ and $\eta_{V}^{n}=-0.3188$ are analytically fixed from data on nucleon magnetic moments:

$$
\eta_{V}^{p}=\left(\frac{\kappa}{M_{N}}\right)^{2}\left(\mu_{p}-1\right), \quad \eta_{V}^{n}=\left(\frac{\kappa}{M_{N}}\right)^{2} \mu_{n},
$$

where $\mu_{p}=2.793$ n.m. and $\mu_{n}=-1.913$ n.m. [34].

The other parameters are fixed from a best fit to data on momentum dependence of electromagnetic nucleon form factors and Roper-nucleon transition helicity amplitudes. In particular, the set on the nucleon parameters

$$
\begin{array}{ll}
g_{V}^{p}=-2.001, & g_{V}^{n}=1.731, \\
\zeta_{V}^{p}=-0.109, & \zeta_{V}^{n}=0.101, \\
\xi_{V}^{p}=-0.166, & \xi_{V}^{n}=0.174, \\
\lambda_{V}^{p}=-0.0005, & \lambda_{V}^{n}=0.0012
\end{array}
$$

is fixed from data on electromagnetic radii and form factors of nucleons. The set of Roper-nucleon parameters

$$
\begin{aligned}
c_{3}^{\mathcal{R} N} & =0.142, \quad c_{4}^{\mathcal{R} N}=-3.942, \quad c_{5}^{\mathcal{R} N}=3.449, \\
g_{V}^{\mathcal{R}_{p} p} & =-10.095, \quad \eta_{V}^{\mathcal{R}_{p} p}=-0.551, \\
\zeta_{V}^{\mathcal{R}_{p} p} & =0.020, \quad \xi_{V}^{\mathcal{R}_{p} p}=-0.770
\end{aligned}
$$

is fixed from data on Roper-nucleon transition data. For simplicity we put $\lambda_{V}^{\mathcal{R}_{p} p}=0$. Our results for quark and nucleon electromagnetic form factors are shown in Figs. 1-9. We compare our results with data [35-84] and the dipole fit $G_{D}\left(Q^{2}\right)=1 /\left(1+Q^{2} / \Lambda^{2}\right)^{2}$. As scale parameter $\Lambda$ we use two values $\Lambda=\sqrt{0.71} \mathrm{GeV}$ and $\Lambda=\sqrt{0.66} \mathrm{GeV}$, corresponding to the root-mean-square (rms) radius $r_{p}=0.81 \mathrm{fm}$ and $r_{p}=0.84 \mathrm{fm}$, respectively. In particular, in Fig. 1 and 2 we present our results for the Dirac and Pauli $u$ (left panel) and $d$ (right panel) quark form factors. Here data are taken from Refs. [35,36].

In Fig. 3 we display the Dirac proton form factor multiplied by $Q^{4}$ (left panel) and the ratio $Q^{2} F_{2}^{p}\left(Q^{2}\right) / F_{1}^{p}\left(Q^{2}\right)$ (right panel). In Fig. 4 we show results for the Dirac neutron form factor multiplied by $Q^{4}$ (left panel) and ratio $\mu_{p} G_{E}^{p}\left(Q^{2}\right) / G_{M}^{p}\left(Q^{2}\right)$ in comparison with global fits (Fit I and Fit II) proposed in Ref. [37]. In our analysis we take the central values of the results for a global fit of the charge and magnetic proton form factors from Ref. [37].

Fit I:

$$
\begin{aligned}
G_{E}^{p}\left(Q^{2}\right) & =\frac{1+a_{1}^{E} \tau}{1+b_{1}^{E} \tau+c_{1}^{E} \tau^{2}+d_{1}^{E} \tau^{3}}, \\
G_{M}^{p}\left(Q^{2}\right) & =\frac{1+a_{1}^{M} \tau}{1+b_{1}^{M} \tau+c_{1}^{M} \tau^{2}+d_{1}^{M} \tau^{3}},
\end{aligned}
$$



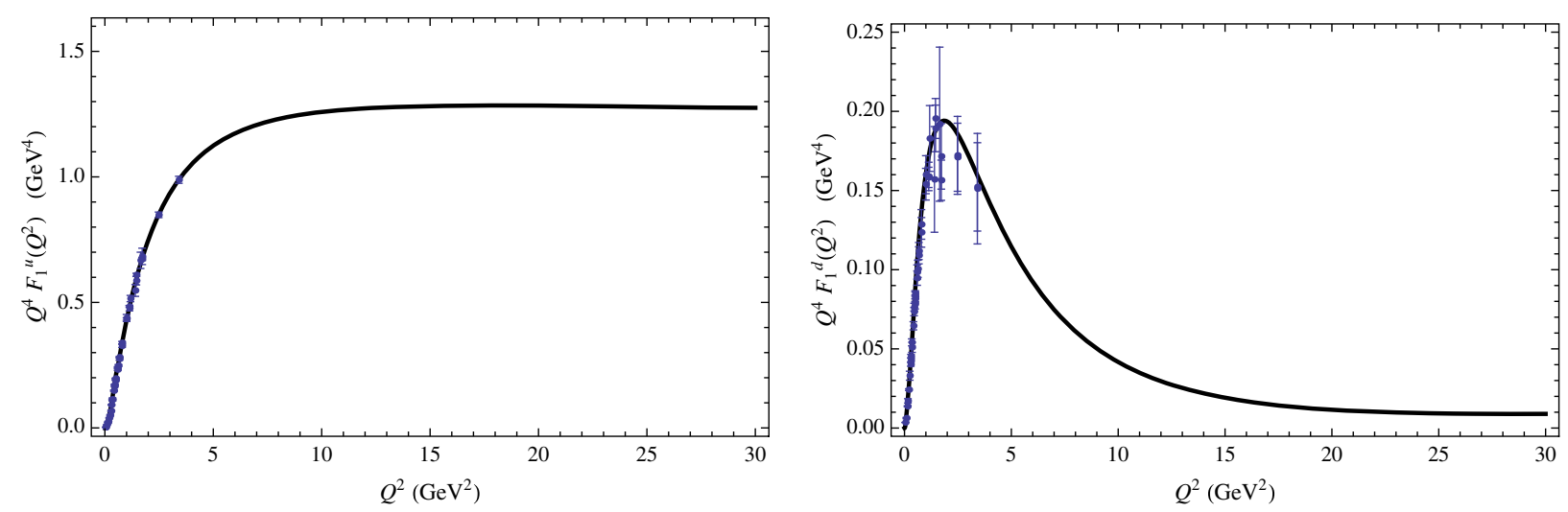

FIG. 1. Dirac $u$ and $d$ quark form factors multiplied by $Q^{4}$.
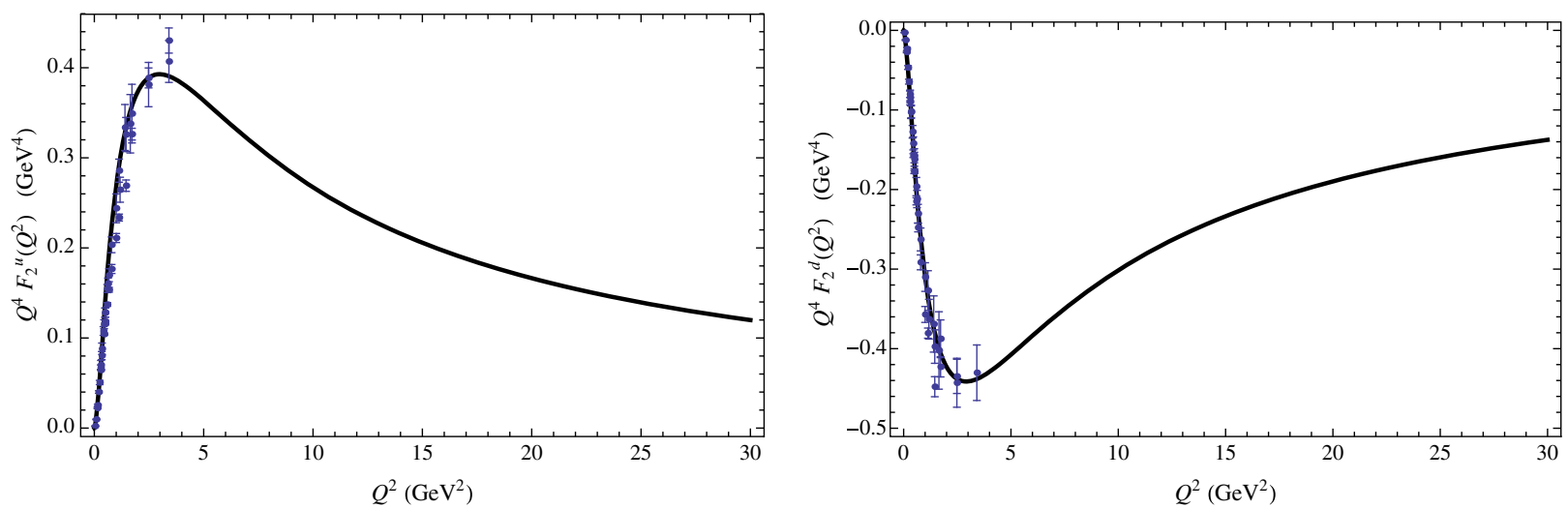

FIG. 2. Pauli $u$ and $d$ quark form factors multiplied by $Q^{4}$.
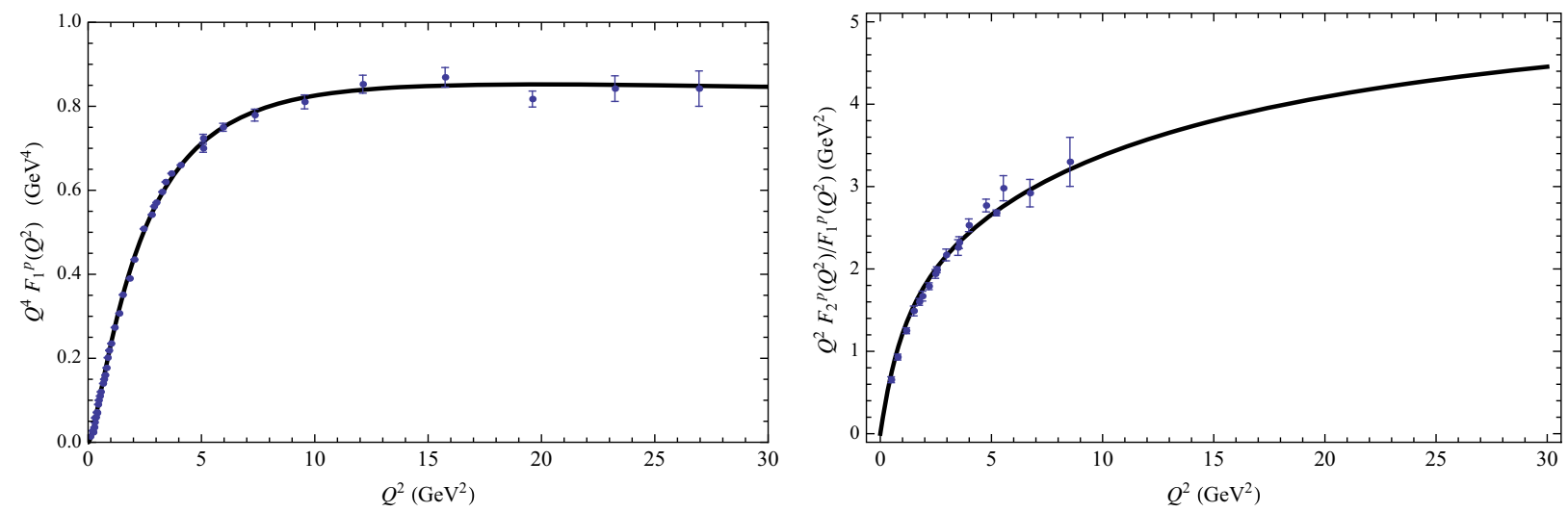

FIG. 3. Dirac proton form factor multiplied by $Q^{4}$ and ratio $Q^{2} F_{2}^{p}\left(Q^{2}\right) / F_{1}^{p}\left(Q^{2}\right)$.

where

$$
\begin{array}{cccc}
a_{1}^{E}=-0.21, & b_{1}^{E}=12.21, & c_{1}^{E}=12.6, & d_{1}^{E}=23.0, \\
a_{1}^{M}=0.058, & b_{1}^{M}=10.85, & c_{1}^{M}=19.9, & d_{1}^{M}=4.4,
\end{array}
$$

Fit II:

$$
\begin{aligned}
G_{E}^{p}\left(Q^{2}\right) & =\frac{1+a_{2}^{E} \tau}{1+b_{2}^{E} \tau+c_{2}^{E} \tau^{2}+d_{2}^{E} \tau^{3}}, \\
G_{M}^{p}\left(Q^{2}\right) & =\frac{1+a_{2}^{M} \tau}{1+b_{2}^{M} \tau+c_{2}^{M} \tau^{2}+d_{2}^{M} \tau^{3}},
\end{aligned}
$$

where 

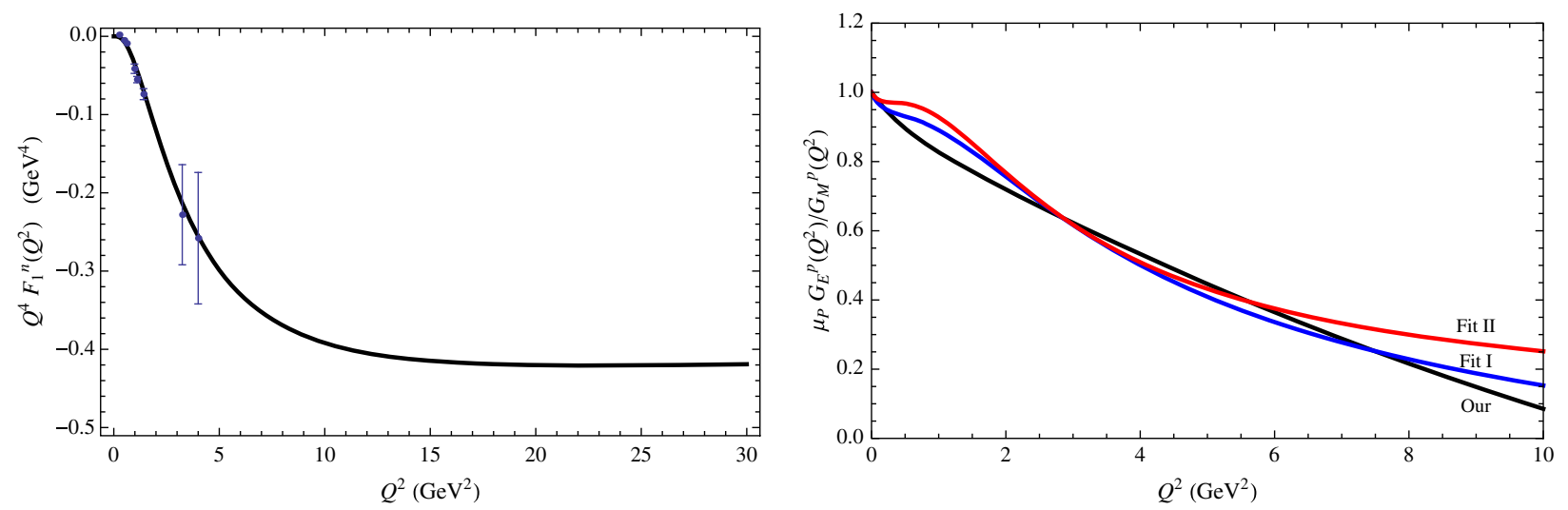

FIG. 4. Dirac neutron form factor multiplied by $Q^{4}$ and ratio $\mu_{p} G_{E}^{p}\left(Q^{2}\right) / G_{M}^{p}\left(Q^{2}\right)$ in comparison with global Fit I and Fit II.
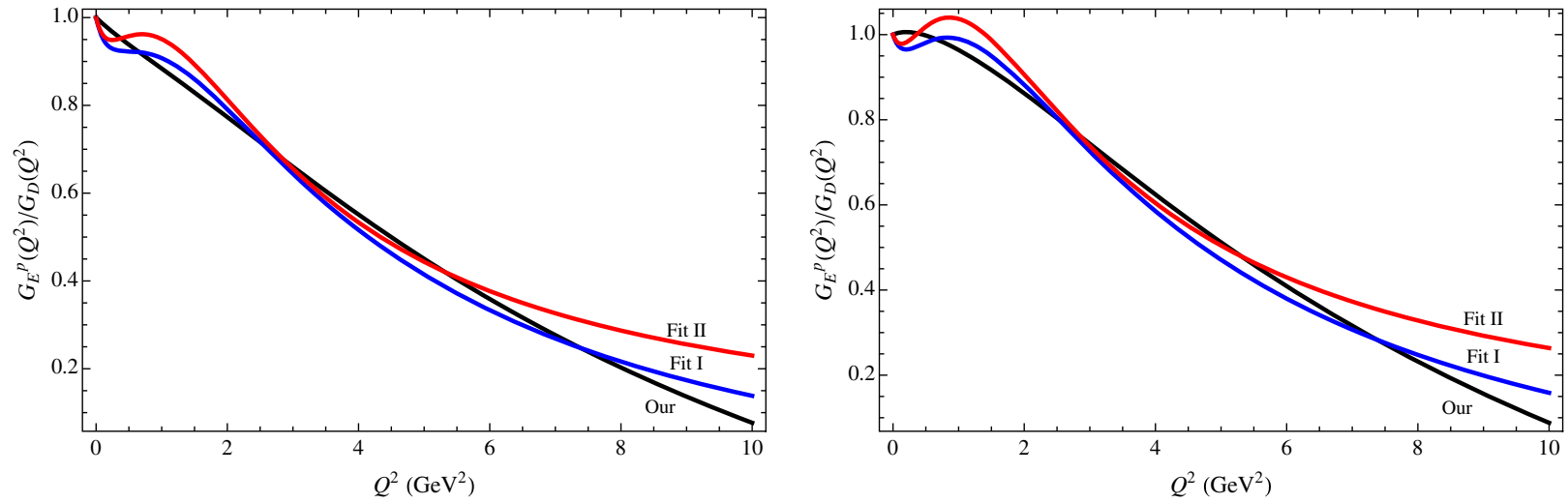

FIG. 5. Ratio $G_{E}^{p}\left(Q^{2}\right) / G_{D}\left(Q^{2}\right)$ in comparison with global Fit I and Fit II for dipole scale parameter $\Lambda^{2}=0.71 \mathrm{GeV}$ (left panel) and $\Lambda^{2}=0.66 \mathrm{GeV}^{2}$ (right panel).
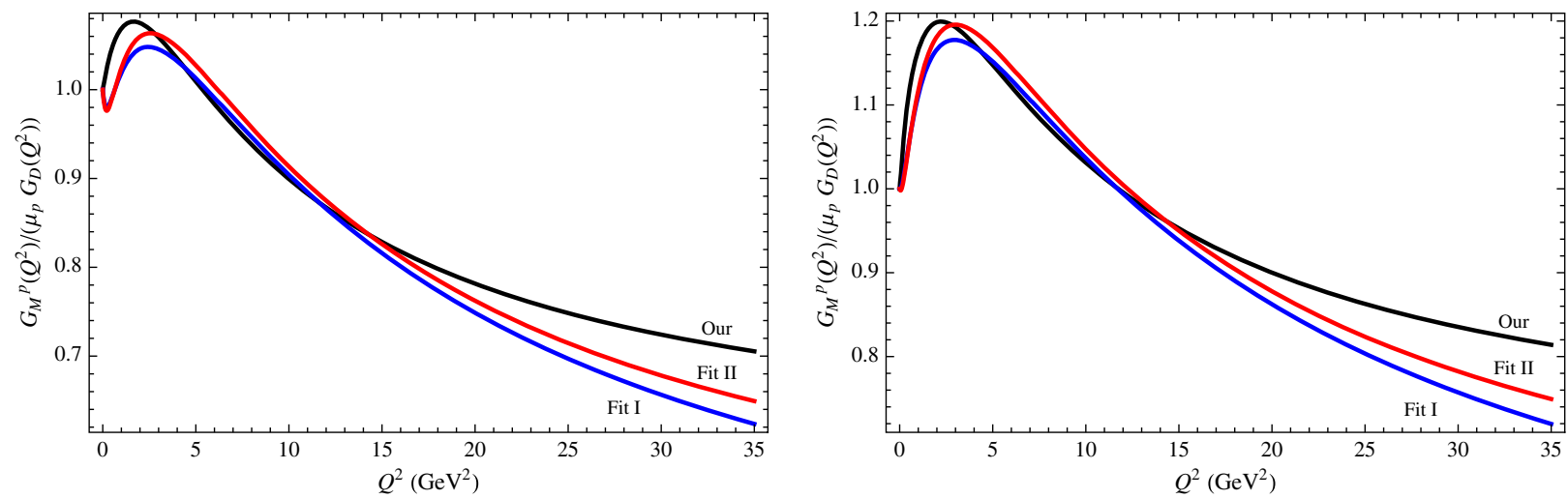

FIG. 6. Ratio $G_{M}^{p}\left(Q^{2}\right) /\left(\mu_{p} G_{D}\left(Q^{2}\right)\right)$ in comparison with global Fit I and Fit II for dipole scale parameter $\Lambda^{2}=0.71 \mathrm{GeV}{ }^{2}$ (left panel) and $\Lambda^{2}=0.66 \mathrm{GeV}^{2}$ (right panel).

$a_{2}^{E}=-0.01, \quad b_{2}^{E}=12.16, \quad c_{2}^{E}=9.7, \quad d_{2}^{E}=37.0$,

$a_{2}^{M}=0.093, \quad b_{2}^{M}=11.07, \quad c_{2}^{M}=19.1, \quad d_{2}^{M}=5.6$.

Here $\tau=Q^{2} /\left(4 M_{N}^{2}\right)$.
In Figs. 5 and 6 we present the ratios $G_{E}^{p}\left(Q^{2}\right) / G_{D}\left(Q^{2}\right)$ and $G_{M}^{p}\left(Q^{2}\right) /\left(\mu_{p} G_{D}\left(Q^{2}\right)\right)$ in comparison with the global Fit I and Fit II [37] for the dipole scale parameter $\Lambda^{2}=$ $0.71 \mathrm{GeV}^{2}$ (left panel) and $\Lambda^{2}=0.66 \mathrm{GeV}^{2}$ (right panel). A detailed comparison of different ratios of the nucleon Sachs form factors is shown in Fig. 7-9. Here we use the 

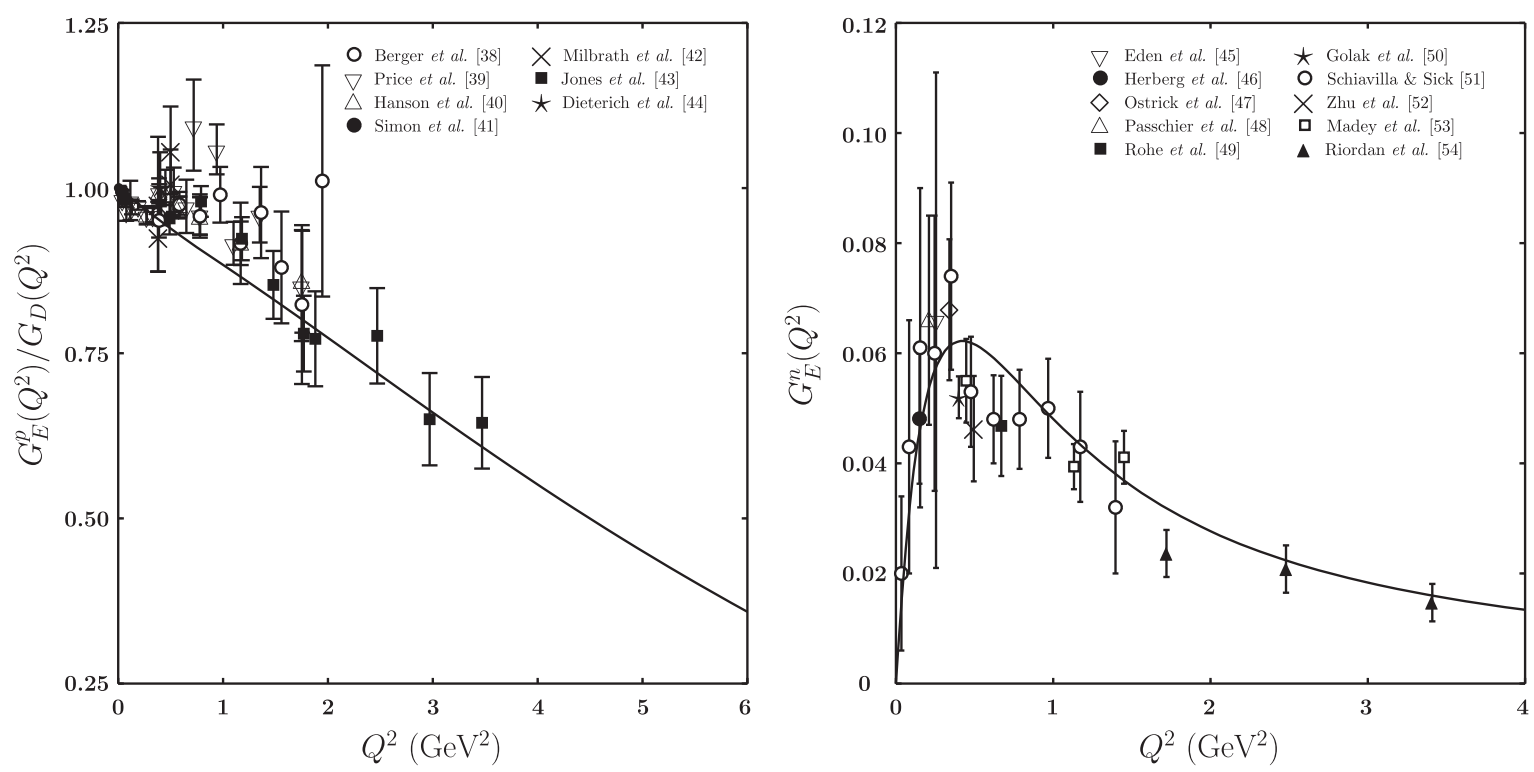

FIG. 7. Ratio $G_{E}^{p}\left(Q^{2}\right) / G_{D}\left(Q^{2}\right)$ and charge neutron form factor $G_{E}^{n}\left(Q^{2}\right)$ in comparison with data.
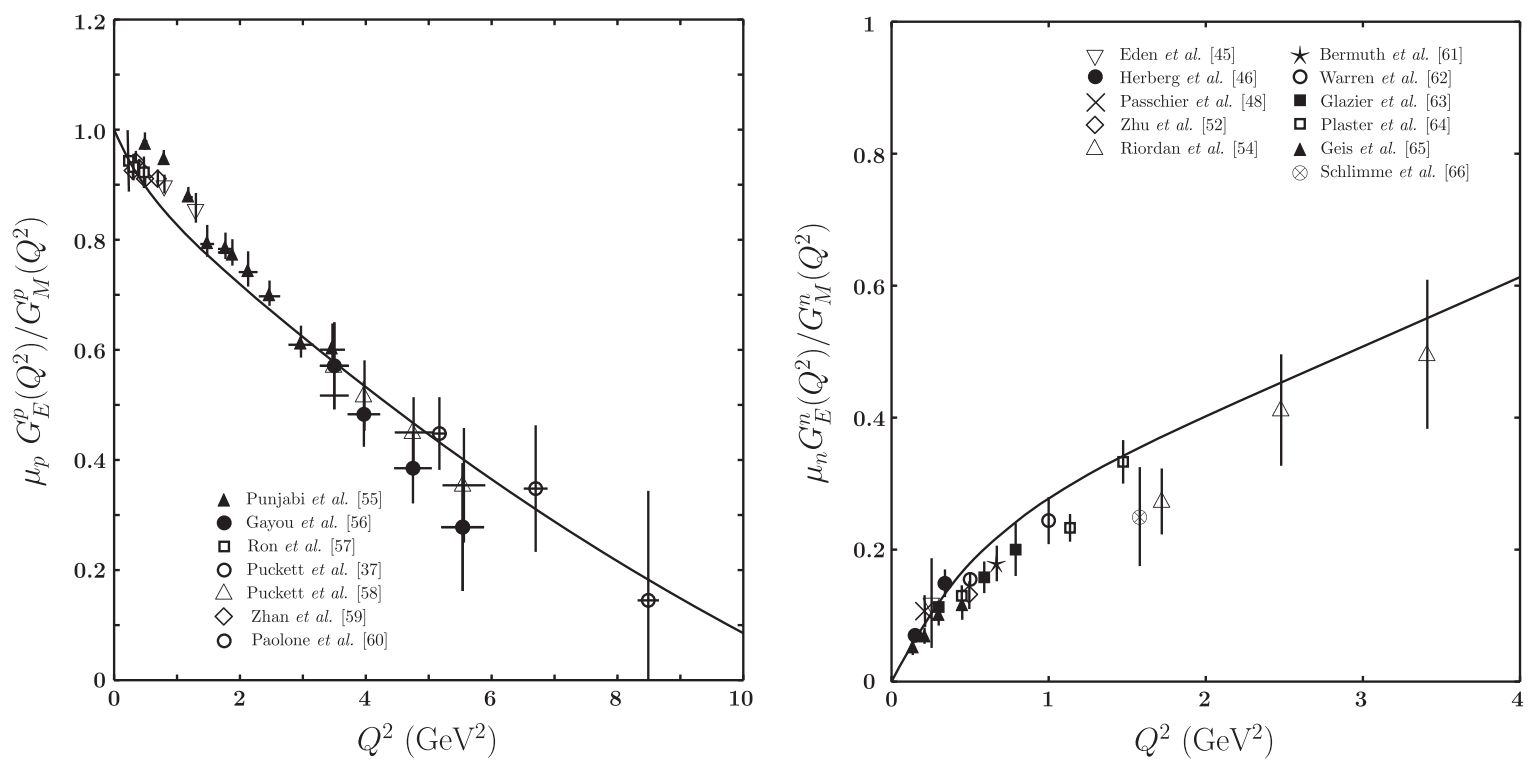

FIG. 8. Ratios $\mu_{p} G_{E}^{p}\left(Q^{2}\right) / G_{M}^{p}\left(Q^{2}\right)$ and $\mu_{n} G_{E}^{n}\left(Q^{2}\right) / G_{M}^{n}\left(Q^{2}\right)$ in comparison with data.

dipole function $G_{D}\left(Q^{2}\right)$ with $\Lambda^{2}=0.71 \mathrm{GeV}^{2}$. The Roper-nucleon transition form factors and helicity amplitudes are shown in Figs. 10 and 11. Our predictions for the Roper-nucleon helicity amplitudes are compared with experimental data of the CLAS (JLab) [23] and A1 (MAMI) [85] Collaborations, and with the MAID parametrization [86]

$$
\begin{aligned}
& A_{1 / 2}^{p}\left(Q^{2}\right)=-0.0614 \mathrm{GeV}^{-1 / 2} \quad\left(1-1.22 \mathrm{GeV}^{-2} Q^{2}-0.55 \mathrm{GeV}^{-8} Q^{8}\right) \exp \left[-1.51 \mathrm{GeV}^{-2} Q^{2}\right], \\
& S_{1 / 2}^{p}\left(Q^{2}\right)=0.0042 \mathrm{GeV}^{-1 / 2} \quad\left(1+40 \mathrm{GeV}^{-2} Q^{2}+1.5 \mathrm{GeV}^{-8} Q^{8}\right) \exp \left[-1.75 \mathrm{GeV}^{-2} Q^{2}\right],
\end{aligned}
$$

and with the parametrization proposed by us. We find that the present data on helicity amplitudes can be fitted with the use of the formulas 

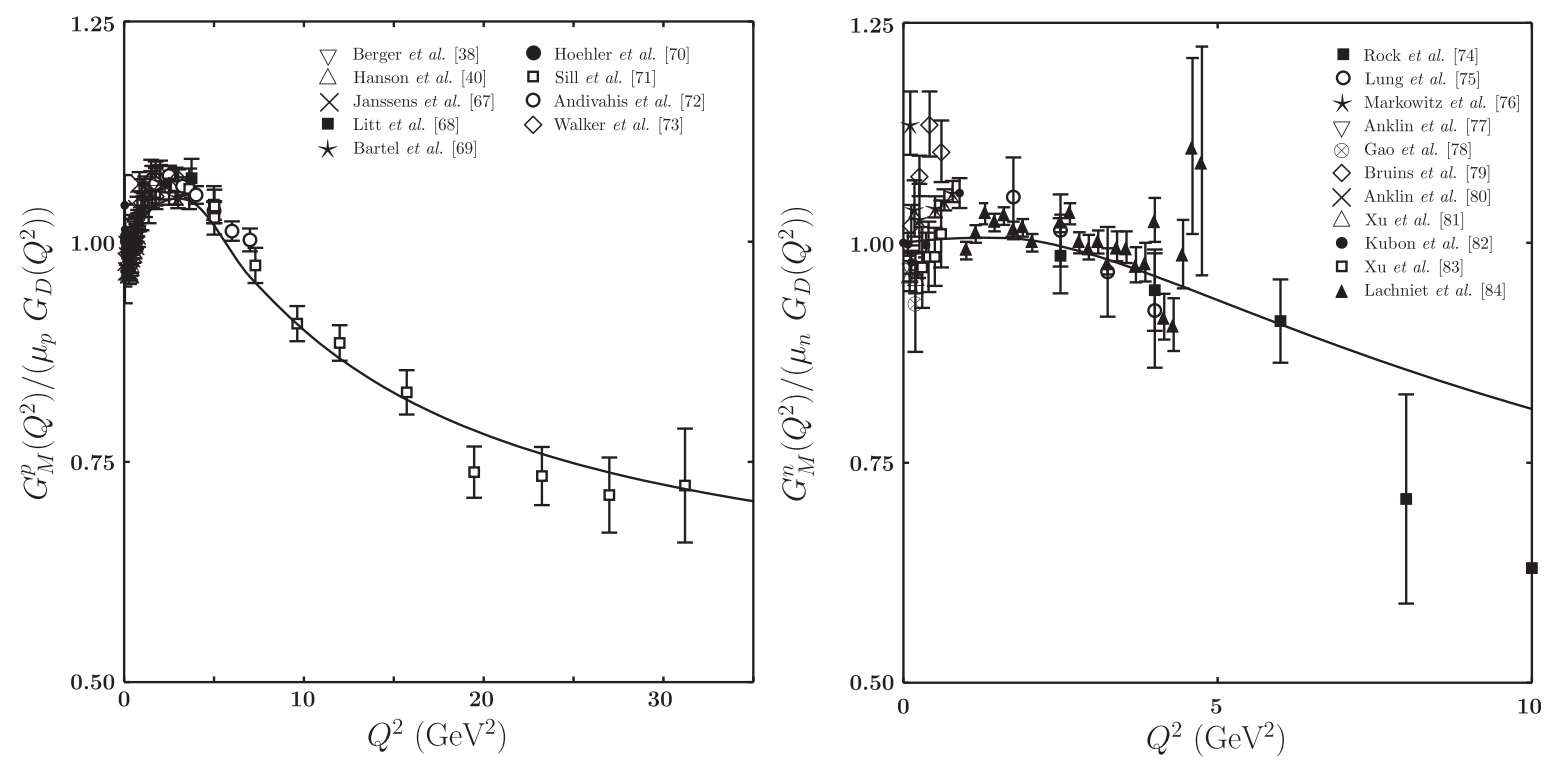

FIG. 9. Ratios $G_{M}^{p}\left(Q^{2}\right) /\left(\mu_{p} G_{D}\left(Q^{2}\right)\right)$ and $G_{M}^{n}\left(Q^{2}\right) /\left(\mu_{n} G_{D}\left(Q^{2}\right)\right)$ in comparison with data.
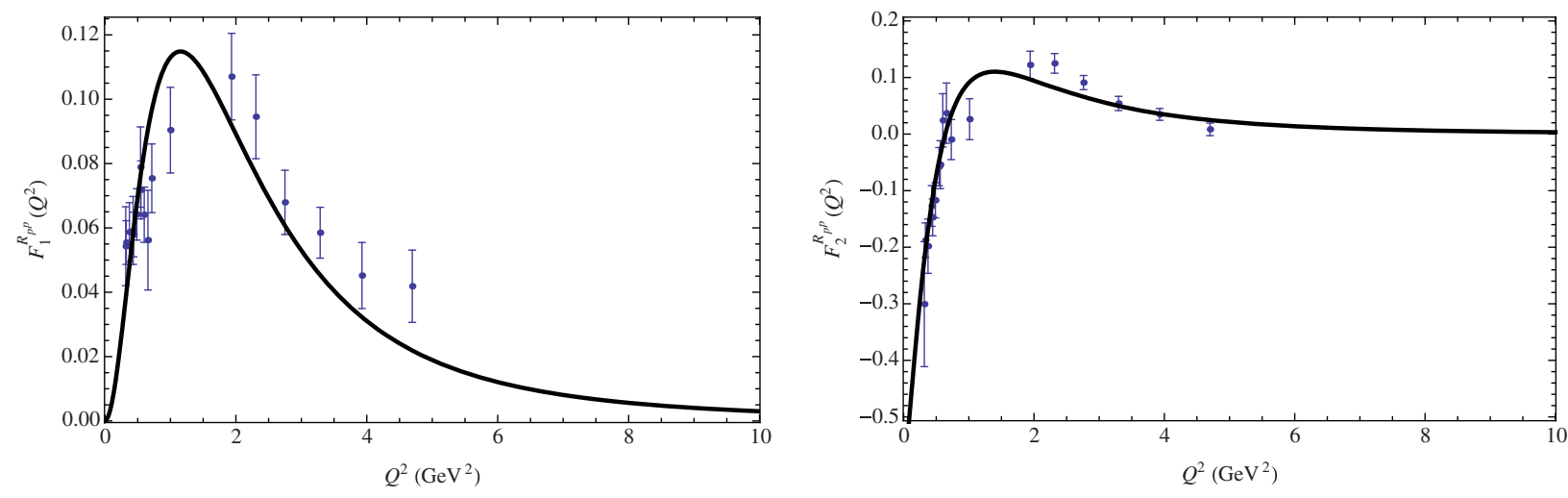

FIG. 10. Roper-nucleon transition form factors $F_{1}^{\mathcal{R}_{p} p}\left(Q^{2}\right)$ and $F_{2}^{\mathcal{R}_{p} p}\left(Q^{2}\right)$ up to $10 \mathrm{GeV}^{2}$.
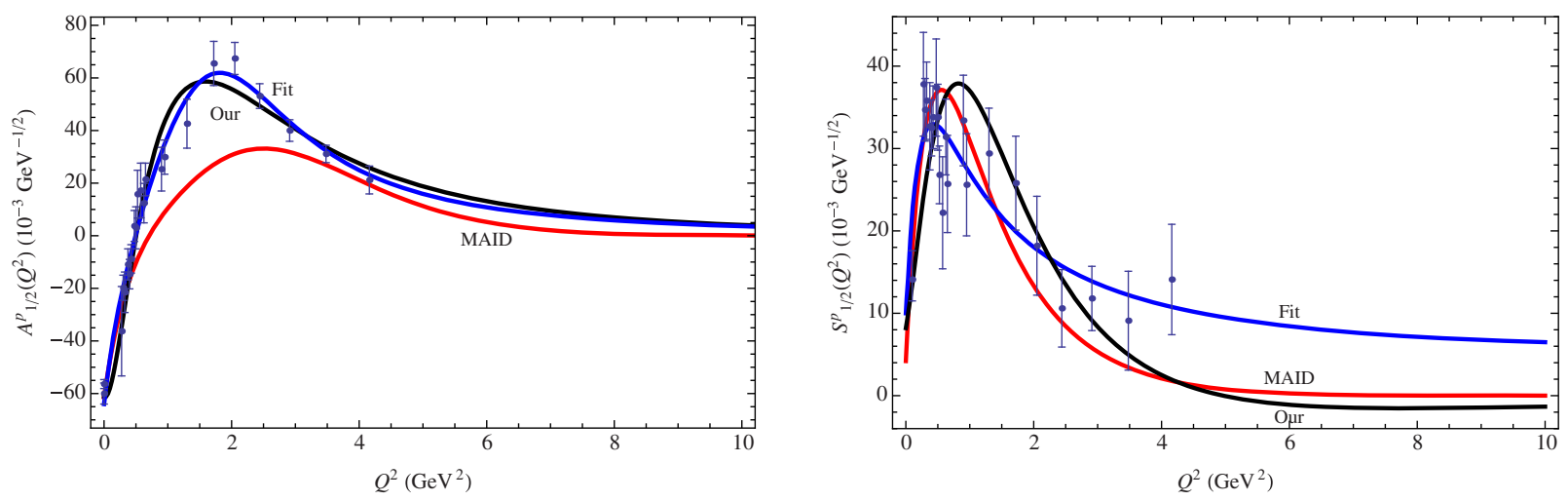

FIG. 11. Helicity amplitudes $A_{1 / 2}^{p}\left(Q^{2}\right)$ and $S_{1 / 2}^{p}\left(Q^{2}\right)$ up to $10 \mathrm{GeV}^{2}$. 
TABLE I. Electromagnetic properties of nucleons and Roper.

\begin{tabular}{lcc}
\hline \hline Quantity & Our results & Data [34] \\
\hline$\mu_{p}$ (in n.m.) & 2.793 & 2.793 \\
$\mu_{n}$ (in n.m.) & -1.913 & -1.913 \\
$r_{E}^{p}(\mathrm{fm})$ & 0.832 & $0.84087 \pm 0.00039$ \\
& & $0.8751 \pm 0.0061$ \\
$\left\langle r_{E}^{2}\right\rangle^{n}\left(\mathrm{fm}^{2}\right)$ & -0.116 & $-0.1161 \pm 0.0022$ \\
$r_{M}^{p}(\mathrm{fm})$ & 0.793 & $0.78 \pm 0.04$ \\
$r_{M}^{n}(\mathrm{fm})$ & 0.813 & $0.864_{-0.008}^{+0.009}$ \\
$A_{1 / 2}^{p}(0)\left(\mathrm{GeV}^{-1 / 2}\right)$ & -0.061 & $-0.060 \pm 0.004$ \\
$S_{1 / 2}^{p}(0)\left(\mathrm{GeV}^{-1 / 2}\right)$ & 0.008 & $\cdots$ \\
\hline \hline
\end{tabular}

$$
\begin{aligned}
& A_{1 / 2}^{p}\left(Q^{2}\right)=A_{1 / 2}^{p}(0) \frac{1+a_{1} Q^{2}}{1+a_{2} Q^{2}+a_{3} Q^{4}+a_{4} Q^{6}}, \\
& S_{1 / 2}^{p}\left(Q^{2}\right)=S_{1 / 2}^{p}(0) \frac{1+s_{1} Q^{2}}{1+s_{2} Q^{2}+s_{3} Q^{4}+s_{4} Q^{6}}
\end{aligned}
$$

where

$$
\begin{gathered}
A_{1 / 2}^{p}(0)=-0.064 \mathrm{GeV}^{-1 / 2}, \\
S_{1 / 2}^{p}(0)=0.010 \mathrm{GeV}^{-1 / 2},
\end{gathered}
$$

and

$$
\begin{array}{ll}
a_{1}=-2.03556 \mathrm{GeV}^{-2}, & a_{2}=1.24891 \mathrm{GeV}^{-2}, \\
a_{3}=-0.90673 \mathrm{GeV}^{-4}, & a_{4}=0.41896 \mathrm{GeV}^{-6}, \\
s_{1}=16.59500 \mathrm{GeV}^{-2}, & s_{2}=1.75908 \mathrm{GeV}^{-2}, \\
s_{3}=3.91487 \mathrm{GeV}^{-4}, & s_{4}=-0.15289 \mathrm{GeV}^{-6} .
\end{array}
$$

Our results for magnetic moments, slope radii and Ropernucleon transition helicity amplitudes at $q^{2}=0$ are summarized in Table I.

\section{SUMMARY}

In the present paper we extended a soft-wall AdS/QCD action by inclusion of novel contributions from additional non-minimal terms. These terms do not renormalize the charge, do not change the normalization of the corresponding form factors and give important contributions to the momentum dependence of the form factors and helicity amplitudes characterizing nucleon and Roper. Extended version of soft-wall AdS/QCD model developed here gives significant improvement of the description of both the nucleon and the Roper structure in comparison with previous versions. In the future we plan to extend our formalism to the study of other nucleon resonances.

\section{ACKNOWLEDGMENTS}

This work was supported by the German Bundesministerium für Bildung und Forschung (BMBF) under Project 05P2015-ALICE at High Rate (BMBF-FSP 202): "Jet- and fragmentation processes at ALICE and the parton structure of nuclei and structure of heavy hadrons", by CONICYT (Chile) Research Project No. 80140097 and under Grants No. 7912010025, No. 1180232 and PIA/ Basal FB0821, by Tomsk State University Competitiveness Improvement Program and the Russian Federation program "Nauka" (Contract No. 0.1764.GZB.2017), and by Tomsk Polytechnic University Competitiveness Enhancement Program (Grant No. VIU-FTI-72/2017).

\section{APPENDIX A: CALCULATION TECHNIQUE}

In this Appendix we briefly discuss calculation technique of electromagnetic form factors of baryons in softwall AdS/QCD model. We illustrate the algorithm for calculating the baryon form factors, considering a particular case of the Dirac form factor of proton $F_{1}\left(Q^{2}\right)$, which is generated by the minimal coupling of vector and fermion AdS fields

$$
\begin{aligned}
S_{\text {int }}= & \int d^{4} x d z \sqrt{g} e^{-\varphi(z)} \\
& \times \sum_{i=+,-; \tau} c_{\tau}^{N} \bar{\psi}_{i, \tau}^{N}(x, z) \Gamma^{M} V_{M}(x, z) \psi_{i, \tau}^{N}(x, z)
\end{aligned}
$$

First we substitute expressions (5)-(7) and (9) for fermion and vector fields into the interaction action (2) and get

$S_{\text {int }}=\int d^{4} x \bar{\psi}_{0}(x) \gamma^{\mu} \psi_{0}(x) \int \frac{d^{4} q}{(2 \pi)^{4}} e^{-i q x} V_{\mu}(q) F_{1}^{p}\left(-q^{2}\right)$

where

$F_{1}^{p}\left(-q^{2}\right)=\frac{1}{2} \sum_{\tau} c_{\tau}^{N} \int_{0}^{\infty} d z V(q, z)\left[\left(f_{\tau, 0}^{L}(z)\right)^{2}+\left(f_{\tau, 0}^{R}(z)\right)^{2}\right]$

is identified with the form factor contributing to the Dirac proton form factor.

The matrix element generating by the action (A2) is

$$
\bar{u}_{p}\left(p_{2}, \lambda_{2}\right) \gamma^{\mu} F_{1}^{p}\left(-q^{2}\right) u_{p}\left(p_{1}, \lambda_{1}\right),
$$

where $u_{p}(p, \lambda)$ is a proton spinor with momentum $p$ and helicity $\lambda ; q=p_{1}-p_{2}$.

Form factor $F_{1}^{p}\left(Q^{2}\right)$ can be presented in terms of the Beta function $B(m, n)=\Gamma(m) \Gamma(n) / \Gamma(m+n)$ after integration over holographic variable $z$. In particular, substituting Eq. (8) and (12) in Eq. (A3) we get 


$$
\begin{aligned}
F_{1}^{p}\left(Q^{2}\right)= & \sum_{\tau} \frac{c_{\tau}^{N}}{\Gamma(\tau)} \int_{0}^{\infty} d z \kappa^{2 \tau} z^{2 \tau-1} \\
& \times \int_{0}^{1} \frac{d x}{(1-x)^{2}} x^{a} e^{-\kappa^{2} z^{2} /(1-x)}\left(\tau-1+\kappa^{2} z^{2}\right) .
\end{aligned}
$$

Changing the variable $k^{2} z^{2} \rightarrow t(1-x)$ we obtain

$$
\begin{aligned}
F_{1}^{p}\left(Q^{2}\right)= & \sum_{\tau} \frac{c_{\tau}^{N}}{2 \Gamma(\tau)} \int_{0}^{1} d x x^{a}(1-x)^{\tau-2} \\
& \times \int_{0}^{\infty} d t t^{\tau-1} e^{-t}(\tau-1+t(1-x)) .
\end{aligned}
$$

Integration over variables $t$ and $x$ is performed using integral representation for Gamma $\Gamma(m)$ and Beta $B(m, n)$ functions, respectively:

$$
\begin{aligned}
\Gamma(m) & =\int_{0}^{\infty} d t t^{m-1} e^{-t}, \\
B(m, n) & =\int_{0}^{1} d x x^{m-1}(1-x)^{n-1} .
\end{aligned}
$$

Finally, we get

$$
F_{1}^{p}\left(Q^{2}\right)=\sum_{\tau} c_{\tau}^{N} B(a+1, \tau)\left(\tau+\frac{a}{2}\right) .
$$

\section{APPENDIX B: THE STRUCTURE INTEGRALS $C_{i}\left(Q^{2}\right), D_{i}\left(Q^{2}\right)$, AND $E_{i}\left(Q^{2}\right)$}

Functions $C_{i}\left(Q^{2}\right), D_{i}\left(Q^{2}\right)$, and $E_{i}\left(Q^{2}\right)$ are given by the analytical expressions

$$
\begin{aligned}
C_{i}\left(Q^{2}\right)= & \sum_{\tau} c_{\tau}^{N} C_{i}^{\tau}\left(Q^{2}\right), \\
C_{1}^{\tau}\left(Q^{2}\right)= & B(a+1, \tau)\left(\tau+\frac{a}{2}\right), \\
C_{2}^{\tau}\left(Q^{2}\right)= & \frac{a}{2} B(a+1, \tau), \\
C_{3}^{\tau}\left(Q^{2}\right)= & a B(a+1, \tau+1) \frac{a(\tau-1)-1}{\tau}, \\
C_{4}^{\tau}\left(Q^{2}\right)= & 2 a[(\tau-1) B(a+1, \tau)-2(2 \tau-1) B(a+1, \tau+1)+3(\tau+1) B(a+1, \tau+2) \\
& \left.+2\left(\tau^{2}-1\right) B(a+2, \tau+1)-2(\tau+1)(\tau+2) B(a+2, \tau+2)\right], \\
C_{5}^{\tau}\left(Q^{2}\right)= & -a[(\tau-1) B(a+1, \tau)+\tau(2 \tau-1) B(a+1, \tau+1)+2 \tau(\tau+1) B(a+1, \tau+2)], \\
C_{6}^{\tau}\left(Q^{2}\right)= & -a[(\tau-1) B(a+1, \tau)+\tau(2 \tau-3) B(a+1, \tau+1)-2 \tau(\tau+1) B(a+1, \tau+2)], \\
C_{7}^{\tau}\left(Q^{2}\right)= & \frac{2 M_{N}}{\kappa}(a+1+\tau) \sqrt{\tau-1} B(a+1, \tau+1), \\
C_{8}^{\tau}\left(Q^{2}\right)= & \frac{4 M_{N}}{\kappa} a \tau \sqrt{\tau-1}[B(a+1, \tau+1)+2(\tau+1) B(a+1, \tau+2)],
\end{aligned}
$$

$$
\begin{aligned}
D_{i}\left(Q^{2}\right)= & \sum_{\tau} c_{\tau}^{\mathcal{R N}} D_{i}^{\tau}\left(Q^{2}\right) \\
D_{1}^{\tau}\left(Q^{2}\right)= & \frac{a}{2} B(a+1, \tau+1)\left[\sqrt{\tau-1}\left(1+\frac{a+1}{\tau}\right)+\sqrt{\tau}\right] \\
D_{2}^{\tau}\left(Q^{2}\right)= & \frac{a}{2} B(a+1, \tau+1)\left[\sqrt{\tau-1}\left(1+\frac{a+1}{\tau}\right)-\sqrt{\tau}\right] \\
D_{3}^{\tau}\left(Q^{2}\right)= & a\left[(\tau-1)^{3 / 2} B(a+1, \tau)-\tau(\sqrt{\tau}+\sqrt{\tau-1}) B(a+1, \tau+1)+(\tau+1) \sqrt{\tau} B(a+1, \tau+2)\right] \\
D_{4}^{\tau}\left(Q^{2}\right)= & 2 a\left[\tau(\tau-1)^{3 / 2} B(a+1, \tau+1)+\tau^{3 / 2}((\tau-2) \sqrt{\tau(\tau-1)}-\tau-1) B(a+1, \tau+2)-(\tau+1) \sqrt{\tau(\tau-1)}(2(\tau+1)\right. \\
& \times \sqrt{\tau-1}+(4 \tau-1) \sqrt{\tau}) B(a+1, \tau+3)+(\tau+1)(\tau+2) \sqrt{\tau}(3+4 \tau+2 \sqrt{\tau(\tau-1)}) B(a+1, \tau+4) \\
& -2(\tau+1)(\tau+2)(\tau+3) \sqrt{\tau} B(a+1, \tau+5)] \\
& \left.+\sqrt{\tau}(\sqrt{\tau}-\sqrt{\tau-1})^{2}(\tau+1) B(a+1, \tau+2)-2 \sqrt{\tau}(\tau+1)(\tau+2) B(a+1, \tau+3)\right] \\
D_{5}^{\tau}\left(Q^{2}\right)= & -a\left[(\tau-1)^{3 / 2} B(a+1, \tau)+\tau(\sqrt{\tau}+\sqrt{\tau-1}(2 \tau-3)) B(a+1, \tau+1)\right.
\end{aligned}
$$




$$
\begin{aligned}
D_{6}^{\tau}\left(Q^{2}\right)= & -a\left[(\tau-1)^{3 / 2} B(a+1, \tau)-\tau(\sqrt{\tau}-\sqrt{\tau-1}(2 \tau-3)) B(a+1, \tau+1)\right. \\
& \left.-\sqrt{\tau}(\sqrt{\tau}+\sqrt{\tau-1})^{2}(\tau+1) B(a+1, \tau+2)+2 \sqrt{\tau}(\tau+1)(\tau+2) B(a+1, \tau+3)\right], \\
D_{7}^{\tau}\left(Q^{2}\right)= & \frac{M_{N}+M_{\mathcal{R}}}{2 \kappa} B(a+1, \tau+1)[a(\tau-1)-\tau-1+a \sqrt{\tau(\tau-1)}], \\
D_{8}^{\tau}\left(Q^{2}\right)= & \frac{M_{N}+M_{\mathcal{R}}}{\kappa} a \sqrt{\tau}(\sqrt{\tau}+\sqrt{\tau-1})[\sqrt{\tau(\tau-1)} B(a+1, \tau+1) \\
& +(\tau+1)(2 \sqrt{\tau(\tau-1)}-1) B(a+1, \tau+2)-2(\tau+1)(\tau+2) B(a+1, \tau+3)],
\end{aligned}
$$

and

$$
\begin{aligned}
E_{i}\left(Q^{2}\right)= & \sum_{\tau} c_{\tau}^{\mathcal{R}} E_{i}^{\tau}\left(Q^{2}\right), \\
E_{1}^{\tau}\left(Q^{2}\right)= & \frac{1}{2}\left[(\tau-1)^{2} B(a+1, \tau-1)+\tau(2-\tau) B(a+1, \tau)-\tau(\tau+1) B(a+1, \tau+1)+(\tau+1)(\tau+2) B(a+1, \tau+2)\right], \\
E_{2}^{\tau}\left(Q^{2}\right)= & \frac{1}{2}\left[(\tau-1)^{2} B(a+1, \tau-1)+\tau(2-3 \tau) B(a+1, \tau)+3 \tau(\tau+1) B(a+1, \tau+1)-(\tau+1)(\tau+2) B(a+1, \tau+2)\right], \\
E_{3}^{\tau}\left(Q^{2}\right)= & a\left[(\tau-1)^{2} B(a+1, \tau)+\tau(2-3 \tau) B(a+1, \tau+1)+3 \tau(\tau+1) B(a+1, \tau+2)-(\tau+1)(\tau+2) B(a+1, \tau+3)\right], \\
E_{4}^{\tau}\left(Q^{2}\right)= & 2 a(\tau+1)\left[\tau(\tau-1)^{2} B(a+1, \tau+2)+\tau\left(2 \tau^{3}-6 \tau^{2}-4 \tau+5\right) B(a+1, \tau+3)-\tau(\tau+2)\left(8 \tau^{2}-2 \tau-13\right) B(a+1, \tau+4)\right. \\
& +(\tau+2)(\tau+3)\left(12 \tau^{2}+10 \tau-4\right) B(a+1, \tau+5)-(8 \tau+7)(\tau+2)(\tau+3)(\tau+4) B(a+1, \tau+6) \\
& +2(\tau+2)(\tau+3)(\tau+4)(\tau+5) B(a+1, \tau+7)], \\
E_{5}^{\tau}\left(Q^{2}\right)= & -a[(\tau-1) B(a+1, \tau)+2 \tau(\tau-1) B(a+1, \tau+1)-(\tau+1) B(a+1, \tau+2)-2(\tau+1)(\tau+2) B(a+1, \tau+3)], \\
E_{6}^{\tau}\left(Q^{2}\right)= & -a[(\tau-1) B(a+1, \tau)+2 \tau(\tau-2) B(a+1, \tau+1)-(\tau+1)(4 \tau-1) B(a+1, \tau+2) \\
& +2(\tau+1)(\tau+2) B(a+1, \tau+3)], \\
E_{7}^{\tau}\left(Q^{2}\right)= & \frac{2 M_{\mathcal{R}}}{\kappa} \sqrt{\tau}[\tau(\tau-1) B(a+1, \tau)-(\tau+1)(2 \tau-1) B(a+1, \tau+1)+(\tau+1)(\tau+2) B(a+1, \tau+2)], \\
E_{8}^{\tau}\left(Q^{2}\right)= & \frac{4 M_{\mathcal{R}}}{\kappa} a \sqrt{\tau}(\tau+1)\left[\tau(\tau-1) B(a+1, \tau+2)+\left(2 \tau^{2}-4 \tau+1\right)(\tau+2) B(a+1, \tau+3)\right. \\
& +(3-4 \tau)(\tau+2)(\tau+3) B(a+1, \tau+4)+2(\tau+2)(\tau+3)(\tau+4) B(a+1, \tau+5)] .
\end{aligned}
$$

[1] Z. Abidin and C. E. Carlson, Nucleon electromagnetic and gravitational form factors from holography, Phys. Rev. D 79, 115003 (2009).

[2] A. Vega, I. Schmidt, T. Gutsche, and V. E. Lyubovitskij, Generalized parton distributions in AdS/QCD, Phys. Rev. D 83, 036001 (2011).

[3] T. Gutsche, V. E. Lyubovitskij, I. Schmidt, and A. Vega, Nucleon structure including high Fock states in AdS/QCD, Phys. Rev. D 86, 036007 (2012).

[4] S. J. Brodsky and G. R. Farrar, Scaling Laws at Large Transverse Momentum, Phys. Rev. Lett. 31, 1153 (1973); V. A. Matveev, R. M. Muradian, and A. N. Tavkhelidze, Automodellism in the large-angle elastic scattering and structure of hadrons, Lett. Nuovo Cimento Soc. Ital. Fis. 7, 719 (1973).
[5] T. Gutsche, V. E. Lyubovitskij, I. Schmidt, and A. Vega, Dilaton in a soft-wall holographic approach to mesons and baryons, Phys. Rev. D 85, 076003 (2012).

[6] S. J. Brodsky, G. F. de Teramond, H. G. Dosch, and J. Erlich, Light-front holographic QCD and emerging confinement, Phys. Rep. 584, 1 (2015).

[7] R. S. Sufian, G. F. de Teramond, S. J. Brodsky, A. Deur, and H. G. Dosch, Analysis of nucleon electromagnetic form factors from light-front holographic QCD: The spacelike region, Phys. Rev. D 95, 014011 (2017).

[8] D. Chakrabarti and C. Mondal, Nucleon and flavor form factors in a light front quark model in AdS/QCD, Eur. Phys. J. C 73, 2671 (2013).

[9] T. Gutsche, V. E. Lyubovitskij, I. Schmidt, and A. Vega, Light-front quark model consistent with Drell-Yan-West 
duality and quark counting rules, Phys. Rev. D 89, 054033 (2014); Erratum, Phys. Rev. D 92, 019902(E) (2015).

[10] T. Gutsche, V.E. Lyubovitskij, I. Schmidt, and A. Vega, Nucleon structure in a light-front quark model consistent with quark counting rules and data, Phys. Rev. D 91, 054028 (2015).

[11] T. Gutsche, V. E. Lyubovitskij, and I. Schmidt, Nucleon parton distributions in a light-front quark model, Eur. Phys. J. C 77, 86 (2017).

[12] V. N. Gribov and L. N. Lipatov, Deep inelastic e p scattering in perturbation theory, Yad. Fiz. 15, 781 (1972) [Sov. J. Nucl. Phys. 15, 438 (1972)]; V. N. Gribov and L. N. Lipatov, $e^{+} e^{-}$pair annihilation and deep inelastic e $\mathrm{p}$ scattering in perturbation theory, Sov. J. Nucl. Phys. 15, 675 (1972) [Yad. Fiz. 15, 1218 (1972)]; G. Altarelli and G. Parisi, Asymptotic freedom in parton language, Nucl. Phys. B126, 298 (1977); Y. L. Dokshitzer, Calculation of the structure functions for deep inelastic scattering and $e^{+} e^{-}$annihilation by perturbation theory in quantum chromodynamics., Sov. Phys. JETP 46, 641 (1977) [Zh. Eksp. Teor. Fiz. 73, 1216 (1977)].

[13] S. J. Brodsky, R. F. Lebed, and V. E. Lyubovitskij, QCD compositeness as revealed in exclusive vector boson reactions through double-photon annihilation: $e^{+} e^{-} \rightarrow \gamma \gamma^{*} \rightarrow$ $\gamma V^{0}$ and $e^{+} e^{-} \rightarrow \gamma^{*} \gamma^{*} \rightarrow V^{0} V^{0}$, Phys. Lett. B 764, 174 (2017).

[14] G. F. de Teramond and S. J. Brodsky, Excited baryons in holographic QCD, AIP Conf. Proc. 1432, 168 (2012).

[15] T. Gutsche, V. E. Lyubovitskij, I. Schmidt, and A. Vega, Nucleon resonances in AdS/QCD, Phys. Rev. D 87, 016017 (2013).

[16] G. Ramalho and D. Melnikov, Valence quark contributions for the $\gamma^{*} N \rightarrow N(1440)$ form factors from light-front holography, arXiv:1703.03819 [Phys. Rev. D (to be published)].

[17] G. Ramalho, Analytic parametrizations of the $\gamma^{*} N \rightarrow$ $N(1440)$ form factors inspired by light-front holography, Phys. Rev. D 96, 054021 (2017).

[18] I. G. Aznauryan et al., Studies of nucleon resonance structure in exclusive meson electroproduction, Int. J. Mod. Phys. E 22, 1330015 (2013).

[19] I. T. Obukhovsky, A. Faessler, D. K. Fedorov, T. Gutsche, and V.E. Lyubovitskij, Electroproduction of the Roper resonance on the proton: the role of the three-quark core and the molecular N $\sigma$ component, Phys. Rev. D 84, 014004 (2011).

[20] I. G. Aznauryan and V. D. Burkert, Nucleon electromagnetic form factors and electroexcitation of low lying nucleon resonances in a light-front relativistic quark model, Phys. Rev. C 85, 055202 (2012).

[21] I. G. Aznauryan et al. (CLAS Collaboration), Electroexcitation of nucleon resonances from CLAS data on single pion electroproduction, Phys. Rev. C 80, 055203 (2009).

[22] V. I. Mokeev et al. (CLAS Collaboration), Experimental Study of the $P_{11}(1440)$ and $D_{13}(1520)$ resonances from CLAS data on $e p \rightarrow e^{\prime} \pi^{+} \pi^{-} p^{\prime}$, Phys. Rev. C 86, 035203 (2012).

[23] V. I. Mokeev et al., New results from the studies of the $N(1440) 1 / 2^{+}, N(1520) 3 / 2^{-}$, and $\Delta(1620) 1 / 2^{-}$resonances in exclusive $e p \rightarrow e^{\prime} p^{\prime} \pi^{+} \pi^{-}$electroproduction with the CLAS detector, Phys. Rev. C 93, 025206 (2016).
[24] H. R. Grigoryan and A. V. Radyushkin, Structure of vector mesons in holographic model with linear confinement, Phys. Rev. D 76, 095007 (2007).

[25] A. Kadeer, J. G. Körner, and U. Moosbrugger, Helicity analysis of semileptonic hyperon decays including lepton mass effects, Eur. Phys. J. C 59, 27 (2009).

[26] A. Faessler, T. Gutsche, M. A. Ivanov, J. G. Körner, and V. E. Lyubovitskij, Semileptonic decays of double heavy baryons in a relativistic constituent three-quark model, Phys. Rev. D 80, 034025 (2009).

[27] T. Branz, A. Faessler, T. Gutsche, M. A. Ivanov, J. G. Körner, V. E. Lyubovitskij, and B. Oexl, Radiative decays of double heavy baryons in a relativistic constituent threequark model including hyperfine mixing, Phys. Rev. D 81, 114036 (2010).

[28] T. Gutsche, M. A. Ivanov, J. G. Körner, V. E. Lyubovitskij, V. V. Lyubushkin, and P. Santorelli, Theoretical description of the decays $\Lambda_{b} \rightarrow \Lambda^{(*)}\left(\frac{1}{2}^{ \pm}, \frac{3}{2}^{ \pm}\right)+J / \psi$, Phys. Rev. D 96, 013003 (2017).

[29] H. J. Weber, Electroexcitation of the $N^{*}$ (1440) in the relativistic constituent quark model, Phys. Rev. C 41, 2783 (1990).

[30] I. G. Aznauryan, Electroexcitation of the Roper resonance in relativistic quark models, Phys. Rev. C 76, 025212 (2007); I. G. Aznauryan, V. D. Burkert, and T.-S. H. Lee, On the definitions of the $\gamma^{*} N \rightarrow N^{*}$ helicity amplitudes, arXiv:0810.0997.

[31] L. A. Copley, G. Karl, and E. Obryk, Electroproduction from nucleons in a relativistic quark model, Phys. Rev. D 4, 2844 (1971).

[32] S. Capstick and B.D. Keister, Baryon current matrix elements in a light front framework, Phys. Rev. D 51, 3598 (1995).

[33] L. Tiator and M. Vanderhaeghen, Empirical transverse charge densities in the nucleon-to- $P_{11}(1440)$ transition, Phys. Lett. B 672, 344 (2009).

[34] C. Patrignani et al. (Particle Data Group), Review of Particle Physics, Chin. Phys. C 40, 100001 (2016).

[35] G. D. Cates, C. W. de Jager, S. Riordan, and B. Wojtsekhowski, Flavor Decomposition of the Elastic Nucleon Electromagnetic Form Factors, Phys. Rev. Lett. 106, 252003 (2011).

[36] M. Diehl and P. Kroll, Nucleon form factors, generalized parton distributions and quark angular momentum, Eur. Phys. J. C 73, 2397 (2013); M. Diehl, Generalized parton distributions from form factors, Nucl. Phys. B, Proc. Suppl. 161, 49 (2006).

[37] A. J. R. Puckett et al., Polarization transfer observables in elastic electron-proton scattering at $Q^{2}=2.5,5.2,6.8$, and $8.5 \mathrm{GeV}^{2}$, Phys. Rev. C 96, 055203 (2017).

[38] C. Berger, V. Burkert, G. Knop, B. Langenbeck, and K. Rith, Electromagnetic form factors of the proton at squared four momentum transfers between 10 and $50 \mathrm{fm}^{-2}$, Phys. Lett. 35B, 87 (1971).

[39] L. E. Price, J. R. Dunning, M. Goitein, K. Hanson, T. Kirk, and R. Wilson, Backward-angle electron-proton elastic scattering and proton electromagnetic form factors, Phys. Rev. D 4, 45 (1971).

[40] K. M. Hanson, J. R. Dunning, M. Goitein, T. Kirk, L. E. Price, and R. Wilson, Large angle quasielastic electrondeuteron scattering, Phys. Rev. D 8, 753 (1973). 
[41] G. G. Simon, C. Schmitt, F. Borkowski, and V. H. Walther, Absolute electron proton cross-sections at low momentum transfer measured with a high pressure gas target system, Nucl. Phys. A333, 381 (1980).

[42] B. D. Milbrath et al. (Bates FPP Collaboration), A Comparison of Polarization Observables in Electron Scattering from the Proton and Deuteron, Phys. Rev. Lett. 80, 452 (1998); Erratum, Phys. Rev. Lett. 82, 2221(E) (1999).

[43] M. K. Jones et al. (Jefferson Lab Hall A Collaboration), $G_{E_{p}} / G_{M_{p}}$ Ratio by Polarization Transfer in $\vec{e} p \rightarrow e \vec{p}$, Phys. Rev. Lett. 84, 1398 (2000).

[44] S. Dieterich et al., Polarization transfer in the ${ }^{4} \mathrm{He}\left(\vec{e}, e^{\prime} \vec{p}\right)^{3} \mathrm{H}$ reaction, Phys. Lett. B 500, 47 (2001).

[45] T. Eden et al., Electric form-factor of the neutron from the ${ }^{2} \mathrm{H}\left(\vec{e}, e^{\prime} \vec{n}\right)^{1} \mathrm{H}$ reaction at $Q^{2}=0.255(\mathrm{GeV} / c)^{2}$, Phys. Rev. C 50, R1749 (1994).

[46] C. Herberg et al., Determination of the neutron electric form factor in the $D\left(e, e^{\prime} n\right) p$ reaction and the influence of nuclear binding, Eur. Phys. J. A 5, 131 (1999).

[47] M. Ostrick et al., Measurement of the Neutron Electric Form Factor $G_{E, n}$ in the Quasifree ${ }^{2} \mathrm{H}\left(\vec{e}, e^{\prime} \vec{n}\right) p$ Reaction, Phys. Rev. Lett. 83, 276 (1999).

[48] I. Passchier et al., Charge Form Factor of the Neutron from the Reaction ${ }^{2} \overrightarrow{\mathrm{H}}\left(\vec{e}, e^{\prime} n\right) p$, Phys. Rev. Lett. 82, 4988 (1999).

[49] D. Rohe et al., Measurement of the Neutron Electric Form Factor $G_{\text {en }}$ at $0.67(\mathrm{GeV} / c)^{2}$ via ${ }^{3} \mathrm{He}\left(\vec{e}, e^{\prime} n\right)$, Phys. Rev. Lett. 83, 4257 (1999).

[50] J. Golak, G. Ziemer, H. Kamada, H. Witala, and W. Glöckle, Extraction of electromagnetic neutron form factors through inclusive and exclusive polarized electron scattering on polarized ${ }^{3} \mathrm{He}$ target, Phys. Rev. C 63, 034006 (2001).

[51] R. Schiavilla and I. Sick, Neutron charge form factor at large $q^{2}$, Phys. Rev. C 64, 041002 (2001).

[52] H. Zhu et al., Measurement of the Electric Form Factor of the Neutron through $\vec{d}\left(\vec{e}, e^{\prime} n\right) p$ at $Q^{2}=0.5(\mathrm{GeV} / c)^{2}$, Phys. Rev. Lett. 87, 081801 (2001).

[53] R. Madey et al. (Jefferson Lab E93-038 Collaboration), Measurements of $G_{E}^{n} / G_{M}^{n}$ from the ${ }^{2} \mathrm{H}\left(\vec{e}, e^{\prime} \vec{n}\right)^{1} \mathrm{H}$ Reaction to $Q^{2}=1.45(\mathrm{GeV} / c)^{2}$, Phys. Rev. Lett. 91, 122002 (2003).

[54] S. Riordan et al., Measurements of the Electric Form Factor of the Neutron up to $Q^{2}=3.4 \mathrm{GeV}^{2}$ Using the Reaction ${ }^{3} \mathrm{He}\left(\vec{e}, e^{\prime} n\right) p p$, Phys. Rev. Lett. 105, 262302 (2010).

[55] V. Punjabi et al., Proton elastic form factor ratios to $Q^{2}=$ $3.5 \mathrm{GeV}^{2}$ by polarization transfer, Phys. Rev. C 71, 055202 (2005); Erratum, Phys. Rev. C 71, 069902(E) (2005).

[56] O. Gayou et al. (Jefferson Lab Hall A Collaboration), Measurement of $G_{E_{p}} / G_{M_{p}}$ in $\vec{e} p \rightarrow e \vec{p} \quad$ to $Q^{2}=5.6 \mathrm{GeV}^{2}$, Phys. Rev. Lett. 88, 092301 (2002).

[57] G. Ron et al. (The Jefferson Lab Hall A Collaboration), Low- $Q^{2}$ measurements of the proton form factor ratio $\mu_{p} G_{E} / G_{M}$, Phys. Rev. C 84, 055204 (2011).

[58] A. J. R. Puckett et al., Final analysis of proton form factor ratio data at $Q^{2}=4.0,4.8$, and $5.6 \mathrm{GeV}^{2}$, Phys. Rev. C 85, 045203 (2012).

[59] X. Zhan et al., High-precision measurement of the proton elastic form factor ratio $\mu_{p} G_{E} / G_{M}$ at low $Q^{2}$, Phys. Lett. B 705, 59 (2011).

[60] M. Paolone et al., Polarization Transfer in the ${ }^{4} \mathrm{He}\left(\vec{e}, e^{\prime} \vec{p}\right)^{3} \mathrm{H}$ Reaction at $Q^{2}=0.8$ and $1.3(\mathrm{GeV} / c)^{2}$, Phys. Rev. Lett. 105, 072001 (2010).
[61] J. Bermuth et al., The neutron charge form factor and target analyzing powers from ${ }^{3} \mathrm{He}\left(\vec{e}, e^{\prime} n\right)$ scattering, Phys. Lett. B 564, 199 (2003).

[62] G. Warren et al. (Jefferson Laboratory E93-026 Collaboration), Measurement of the Electric Form Factor of the Neutron at $Q^{2}=0.5$ and $1.0 \mathrm{GeV}^{2} / c^{2}$, Phys. Rev. Lett. 92, 042301 (2004).

[63] D. I. Glazier et al., Measurement of the electric form factor of the neutron at $Q^{2}=0.3-0.8(\mathrm{GeV} / c)^{2}$, Eur. Phys. J. A 24, 101 (2005).

[64] B. Plaster et al. (Jefferson Laboratory E93-038 Collaboration), Measurements of the neutron electric to magnetic form factor ratio $G_{E n} / G_{M n}$ via the ${ }^{2} \mathrm{H}\left(\vec{e}, e^{\prime} \vec{n}\right)^{1} \mathrm{H}$ reaction to $Q^{2}=1.45(\mathrm{GeV} / c)^{2}$, Phys. Rev. C 73, 025205 (2006).

[65] E. Geis et al. (BLAST Collaboration), Charge Form Factor of the Neutron at Low Momentum Transfer from the ${ }^{3} \overrightarrow{\mathrm{H}}\left(\vec{e}, e^{\prime} n\right){ }^{1} \mathrm{H}$ Reaction, Phys. Rev. Lett. 101, 042501 (2008).

[66] B. S. Schlimme et al., Measurement of the Neutron Electric to Magnetic Form Factor Ratio at $Q^{2}=1.58 \mathrm{GeV}^{2}$ Using the Reaction ${ }^{3} \mathrm{He}\left(\vec{e}, e^{\prime} n\right) p p$, Phys. Rev. Lett. 111, 132504 (2013).

[67] T. Janssens, R. Hofstadter, E. B. Hughes, and M. R. Yearian, Proton form factors from elastic electron-proton scattering, Phys. Rev. 142, 922 (1966).

[68] J. Litt et al., Measurement of the ratio of the proton form factors, $\mathrm{G}_{\mathrm{E}} / \mathrm{G}_{\mathrm{M}}$, at high momentum transfers and the question of scaling, Phys. Lett. 31B, 40 (1970).

[69] W. Bartel, F.-W. Büsser, W.-R. Dix, R. Felst, D. Harms, H. Krehbiel, P. E. Kuhlmann, J. McElroy, J. Meyer, and G. Weber, Measurement of proton and neutron electromagnetic form factors at squared four momentum transfers up to $3(\mathrm{GeV} / c)^{2}$, Nucl. Phys. B58, 429 (1973).

[70] G. Höhler, E. Pietarinen, I. Sabba-Stefanescu, F. Borkowski, G. G. Simon, V. H. Walther, and R. D. Wendling, Analysis of electromagnetic nucleon form factors, Nucl. Phys. B114, 505 (1976).

[71] A.F. Sill et al., Measurements of elastic electron-proton scattering at large momentum transfer, Phys. Rev. D 48, 29 (1993).

[72] L. Andivahis et al., Measurements of the electric and magnetic form factors of the proton from $Q^{2}=1.75$ to 8.83(GeV/c $)^{2}$, Phys. Rev. D 50, 5491 (1994).

[73] R. C. Walker et al., Measurements of the proton elastic form factors for $1 \leq Q^{2} \leq 3(\mathrm{GeV} / c)^{2}$ at SLAC, Phys. Rev. D 49, 5671 (1994).

[74] S. Rock, R. G. Arnold, P. Bosted, B. T. Chertok, B. A. Mecking, I. Schmidt, Z. M. Szalata, R. C. York, and R. Zdarko, Measurement of Elastic Electron-Neutron Cross Sections up to $Q^{2}=10(\mathrm{GeV} / c)^{2}$, Phys. Rev. Lett. 49, 1139 (1982).

[75] A. Lung et al., Measurements of the Electric and Magnetic Form Factors of the Neutron from $Q^{2}=1.75$ to 4.00(GeV/c $)^{2}$, Phys. Rev. Lett. 70, 718 (1993).

[76] P. Markowitz et al., Measurement of the magnetic form factor of the neutron, Phys. Rev. C 48, R5 (1993).

[77] H. Anklin et al., Precision measurement of the neutron magnetic form factor, Phys. Lett. B 336, 313 (1994).

[78] H. Gao et al., Measurement of the neutron magnetic form factor from inclusive quasielastic scattering of polarized electrons from polarized ${ }^{3} \mathrm{He}$, Phys. Rev. C 50, R546 (1994). 
[79] E. E. W. Bruins et al., Measurement of the Neutron Magnetic Form Factor, Phys. Rev. Lett. 75, 21 (1995).

[80] H. Anklin et al., Precise measurements of the neutron magnetic form factor, Phys. Lett. B 428, 248 (1998).

[81] W. Xu et al. The Transverse Asymmetry $A_{T^{\prime}}$ from Quasielastic Polarized ${ }^{3} \mathrm{He}\left(\vec{e}, e^{\prime}\right)$ Process and the Neutron Magnetic Form Factor, Phys. Rev. Lett. 85, 2900 (2000).

[82] G. Kubon et al., Precise neutron magnetic form factors, Phys. Lett. B 524, 26 (2002).

[83] W. Xu et al. (Jefferson Lab E95-001 Collaboration), Planewave impulse approximation extraction of the neutron magnetic form factor from quasielastic ${ }^{3} \mathrm{He} \rightarrow\left(\vec{e}, e^{\prime}\right)$ at $Q^{2}=0.3$ to $0.6(\mathrm{GeV} / c)^{2}$, Phys. Rev. C 67, 012201 (2003).

[84] V. J. Lachniet et al. (CLAS Collaboration), A Precise Measurement of the Neutron Magnetic Form Factor $G_{M}^{n}$ in the Few- $\mathrm{GeV}^{2}$ Region, Phys. Rev. Lett. 102, 192001 (2009).

[85] S. Stajner et al., Beam-Recoil Polarization Measurement of $\pi^{0}$ Electroproduction on the Proton in the Region of the Roper Resonance, Phys. Rev. Lett. 119, 022001 (2017).

[86] D. Drechsel, S. S. Kamalov, and L. Tiator, Unitary isobar model-MAID2007, Eur. Phys. J. A 34, 69 (2007). 